\title{
A New Ensemble Semi-supervised Self-labeled Algorithm
}

\author{
Ioannis Livieris \\ Department of Computer \& Informatics Engineering \\ Technological Educational Institute of Western Greece, Greece, GR 263-34 \\ E-mail: livieris@ teiwest.gr
}

Keywords: semi-supervised methods, self-labeled, ensemble methods, classification, voting

Received: March 13, 2018

\begin{abstract}
As an alternative to traditional classification methods, semi-supervised learning algorithms have become a hot topic of significant research, exploiting the knowledge hidden in the unlabeled data for building powerful and effective classifiers. In this work, a new ensemble-based semi-supervised algorithm is proposed which is based on a maximum-probability voting scheme. The reported numerical results illustrate the efficacy of the proposed algorithm outperforming classical semi-supervised algorithms in term of classification accuracy, leading to more efficient and robust predictive models.
\end{abstract}

Povzetek: Razvit je nov delno nadzorovani učni algoritem s pomočjo ansamblov in glasovalno shemo na osnovi največje verjetnosti.

\section{Introduction}

The development of a powerful and accurate classifier is considered as one of the most significant and challenging tasks in machine learning and data mining [3]. Nevertheless, it is generally recognized that the key to recognition problems does not lie wholly in any particular solution since no single model exists for all pattern recognition problems $[28,15]$.

During the last decades, in the area of machine learning the development of an ensemble of classifiers has been proposed as a new direction for improving the classification accuracy. The basic idea of ensemble learning is the combination of a set of diverse prediction models, each of which solves the same original task, in order to obtain a better composite global model with more accurate and reliable estimates or decisions than can be obtained from using a single model $[9,28]$. Therefore, several prediction models have been proposed based on ensembles techniques which have been successfully utilized to tackle difficult real-world problems [31, 14, 32, 30, 23, 27, 11]. Traditional ensemble methods usually combine the individual predictions of supervised algorithms which utilize only labeled data as training set. However, in most real-world classification problems, the acquisition of sufficient labeled samples is cumbersome and expensive and frequently requires the efforts of domain experts. On the other hand, unlabeled data are fairly easy to obtain and require less effort of experienced human annotators.

Semi-supervised learning algorithms constitute the appropriate and effective machine learning methodology for extracting useful knowledge from both labeled and unlabeled data. In contrast to traditional classification approaches, semi-supervised algorithms utilize a large amount of unlabeled samples to either modify or reprior- itize the hypothesis obtained from labeled samples in order to build an efficient and accurate classifier. The general assumption of these algorithms is to leverage the large amount of unlabeled data in order to reduce data sparsity in the labeled training data and boost the classifier performance, particularly focusing on the setting where the amount of available labeled data is limited. Hence, these methods have received considerable attention due to their potential for reducing the effort of labeling data while still preserving competitive and sometimes better classification performance (see $[18,6,7,38,17,16,21,20,22,44,45$, $46,43]$ and the references therein). The main issue in semi-supervised learning is how to exploit the information hidden in the unlabeled data. In the literature, several approaches have been proposed each with different philosophy related to the link between the distribution of labeled and unlabeled data $[46,4,36]$.

Self-labeled methods constitute semi-supervised methods which address the shortage of labeled data via a selflearning process based on supervised prediction models. The main advantages of this class of methods are their simplicity and their wrapper-based philosophy. The former is related to the facility/comodity of application and implementation while the latter refers to the fact that any supervised classifier can be utilized, independent of its complexity [35]. In the literature, self-labeled methods are divided into self-training [41] and co-training [4]. Self-training constitutes an efficient semi-supervised method which iteratively enlarges the labeled training set by adding the most confident predictions of the utilized supervised classifier. The standard co-training method splits the feature space into two different conditionally independent views. Subsequently, it trains one classifier in each specific view and the classifiers teach each other the most confidently predicted examples. More sophisticated and advanced variants 
of this method do not require explicit feature splits or the iterative mutual-teaching procedure imposed by co-training, as they are commonly based on disagreement-based classifiers [44, 12, 36, 46, 45]

By taking these into consideration, ensemble methods and semi-supervised methods constitute two significant classes of methods. The former attempt to achieve strong classification performance by combining individual classifiers while the later attempt to enhance the performance of a classifier by exploiting the information in the unlabeled data. Although both methodologies have been efficiently applied to a variety of real-world problems during the last decade, they were almost developed separately. In this context, Zhou [43] advocated that ensemble learning and semisupervised learning are indeed beneficial to each other and stronger learning machines can be generated by leveraging unlabeled data with the combination of diverse classifiers. More specifically, ensemble learning could be useful to semi-supervised learning since an ensemble of classifiers could be more accurate than an individual classifier. Additionally, semi-supervised learning could assist ensemble learning since unlabeled data can enhance the diversity of the base learner which constitute the ensemble and increase the ensemble's classification accuracy.

In this work, a new ensemble semi-supervised selflabeled learning algorithm is proposed. The proposed algorithm combines the individual predictions of three of the most representative SSL algorithms: Self-training, Cotraining and Tri-training via a maximum-probability voting scheme. The efficiency of the proposed algorithm is evaluated on various standard benchmark datasets and the reported experimental results illustrate its efficacy in terms of classification accuracy, leading to more efficient and robust prediction models.

The remainder of this paper is organized as follows: Section 3 presents some elementary semi-supervised learning definitions and Section 4 presents a detailed description of the proposed algorithm. Section 5 presents the experimental results of the comparison of the proposed algorithm with the most popular semi-supervised classification methods on standard benchmark datasets. Finally, Section 6 discusses the conclusions and some research topics for future work.

\section{Related work}

Semi-Supervised Learning (SSL) and Ensemble Learning (EL) constitute machine learning techniques which were independently developed to improve the performance of existing learning methods, though from different perspectives and methodologies. SSL provides approaches to improve model generalization performance by exploiting unlabeled data; while EL explores the possibility of achiev- ing the same objective by aggregating a group of learners. Zhou [43] presented an extensive analysis of how semi-supervised learning and ensemble learning can be efficiently fuse for the development of efficient prediction models. A number of rewarding studies which fuse and exploit their advantages have been carried out in recent years; some useful outcomes of them are briefly presented below.

Zhou and Goldman [42] have adopted the idea of ensemble learning and majority voting and proposed a new SSL algorithm which is based on the multi-learning approach. More specifically, this algorithm utilizes multiple algorithms for producing the necessary information and endorses a voted majority process for the final decision, instead of asking for more than one views of the corresponding data.

Along this line, Li and Zhou [17] proposed another algorithm, in which a number of Random trees are trained on bootstrap data from the dataset, named Co-Forest. The main idea of this algorithm is the assignment of a few unlabeled examples to each Random tree during the training process. Eventually, the final decision is composed by a simple majority voting. Notice that the utilization of Random Tree classifier for random samples of the collected labeled data is the main reason why the behavior Co-Forest is efficient and robust although the number of the available labeled examples is reduced. Xu et al. [40] applied this method for the predictions of protein subcellular localization providing some promising results.

Sun and Zhang [34] attempted to combine the advantages of multiple-view learning and ensemble learning for semi-supervised learning. They proposed a novel multiple-view multiple-learner framework for semisupervised learning which adopted a co-training based learning paradigm in enlarging labeled data from a much larger set of unlabeled data. Their motivation is based on the fact that the use of multiple views is promising to promote performance compared with single-view learning because information is more effectively exploited; while at the same time, as an ensemble of classifiers is learned from each view, predictions with higher accuracies can be obtained than solely adopting one classifier from the same view. The experiments conduced on several datasets presented some encouraging results, illustrating the efficacy of the proposed method.

Roy et al. [29] presented a novel approach by utilizing a multiple classifier system in the SSL framework instead of using a single weak classifier for change detection in remotely sensed images. The proposed algorithm during the iterative learning process uses the agreement between all the classifiers which constitute the ensemble for collecting the most confident labeled patterns. The effectiveness of the proposed technique was presented by a variety of experiments carried out on multi-temporal and multi-spectral 
datasets.

In more recent works, Livieris et al. [21] proposed a new ensemble-based semi-supervised method for the prognosis of students' performance in the final examinations. They incorporated a ensemble of classifiers as base learner in the semi-supervised framework. Based on their numerical experiments, the authors concluded that ensemble methods and semi-supervised methodologies could efficiently combined to develop efficient prediction models. Motivated by the previous work, Livieris et al. [22] presented a new ensemble-based semi-supervised learning algorithm for the classification of chest X-rays of tuberculosis, presenting some encouraging results.

\section{A review on semi-supervised self-labeled classification}

In this section, we present a formal definition of the semisupervised classification problem and briefly describe the most relevant self-labeled approaches proposed in the literature. Let $x_{p}=\left(x_{p 1}, x_{p 2}, \ldots, x_{p D}, y\right)$ be an example, where $x_{p}$ belongs to a class $y$ and a $D$-dimensional space in which $x_{p i}$ is the $i$-th attribute of the $p$-th sample. Suppose $L$ is a labeled set of $N_{L}$ instances $x_{p}$ with $y$ known and $U$ is an unlabeled set of $N_{U}$ instance $x_{q}$ with $y$ unknown. Notice that the set $L \cup U$ consists the training set. Moreover, there exists a test set $T$ of $N_{T}$ unseen instances where $y$ is unknown, which has not been utilized in the training stage. Notice that the aim of the semi-supervised classification is to obtain an accurate and robust learning hypothesis with the use of the training set.

Self-labeled techniques constitute a significant family of classification methods which progressively classify unlabeled data based on the most confident predictions and utilize them to modify the hypothesis learned from labeled samples. Therefore, the methods of this class accept that their own predictions tend to be correct, without making any specific assumptions about the input data. In the literature, a variety of self-labeled methods has been proposed each with different philosophy and methodology on exploiting the information hidden in the unlabeled data. In this work, we focus our attention to Selftraining, Co-training and Tri-training which constitute the most efficient and commonly used self-labeled methods $[21,20,22,35,37,36]$.

\subsection{Self-Training}

Self-training [41] is generally considered as the simplest and one of the most efficient SSL algorithms. This algorithm is a wrapper based SSL approach which constitutes an iterative procedure of self-labeling unlabeled data. According to $\mathrm{Ng}$ and Cardie [25] "self-training is a singleview weakly supervised algorithm" which is based on its own predictions on unlabeled data to teach itself. Firstly, an arbitrary classifier is initially trained with a small amount of labeled data, constituting its training set which is iteratively augmented using its own most confident predictions of the unlabeled data. More analytically, each unlabeled instance which has achieved a probability over a specific threshold ConLev is considered sufficiently reliable to be added to the labeled training set and subsequently the classifier is retrained.

Clearly, the success of Self-training is heavily depended on the newly-labeled data based on its own predictions, hence its weakness is that erroneous initial predictions will probably lead the classifier to generate incorrectly labeled data [46]. A high-level description of Self-training algorithm is presented in Algorithm 1.

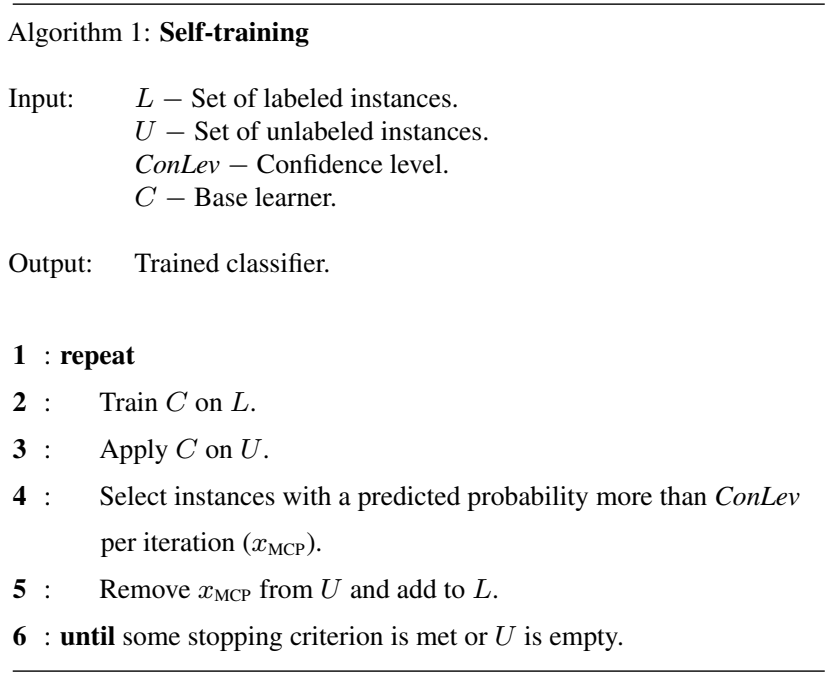

\subsection{Co-training}

Co-training [4] is a SSL algorithm which utilizes two classifiers, each trained on a different view of the labeled training set. The underlying assumptions of the Co-training approach is that feature space can be split into two different conditionally independent views and that each view is able to predict the classes perfectly [33]. Under these assumptions, two classifiers are trained separately for each view using the initial labeled set and then iteratively the classifiers augment the training set of the other with the most confident predictions on unlabeled examples. 
Essentially, Co-training is a "two-view weakly supervised algorithm" since it uses the self-training approach on each view [25]. Blum and Mitchell [4] have extensively studied the efficacy of Co-training and they concluded that if the two views are conditionally independent, then the use of unlabeled data can significantly improve the predictive accuracy of a weak classifier. Nevertheless, the assumption about the existence of sufficient and redundant views is a luxury hardly met in most real world scenarios. Algorithm 2 presents a high-level description of Co-training algorithm.

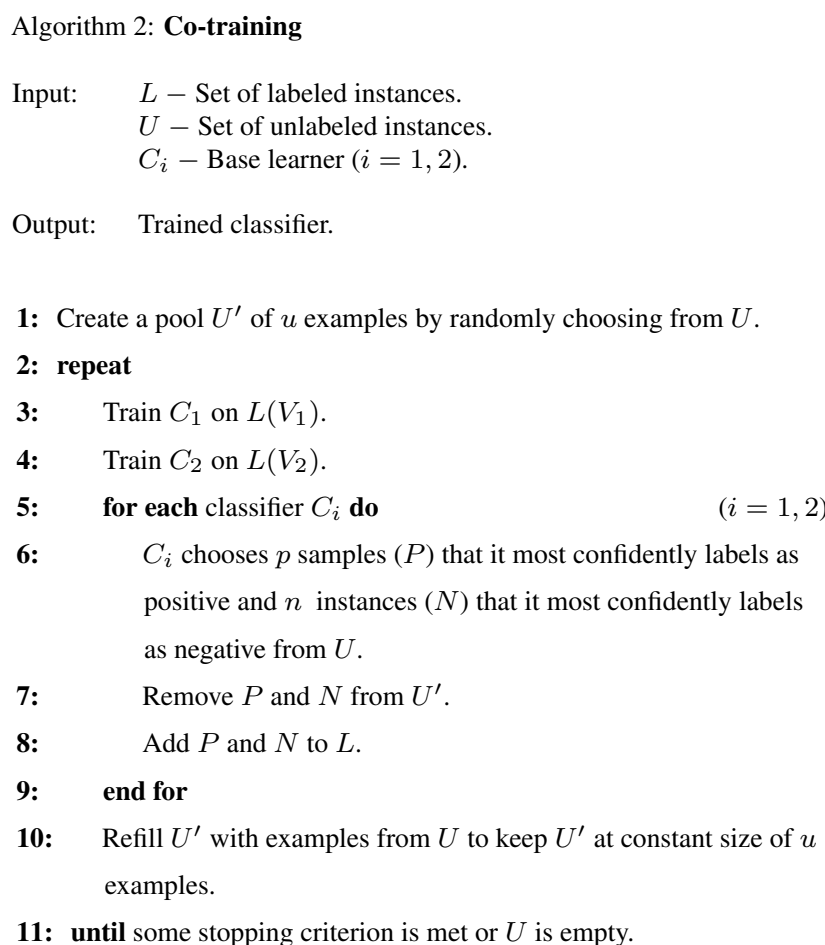

Remark: $V_{1}$ and $V_{2}$ are two feature conditionally independent views of instances.

\subsection{Tri-Training}

Tri-Training [44] consists of an improved version of CoTraining which overcomes the requirements for multiple sufficient an redundant feature sets. This algorithm constitutes a bagging ensemble of three classifiers, trained on the data subsets generated through bootstrap sampling from the original labeled training set. In case two of the three classifiers agree on the categorization of an unlabeled instance, then this is considered to be labeled and augment the third classifier with the newly labeled example. The efficiency of the training process is based on the strategy the "majority teach minority" which avoids the use of a complicated time consuming approach to explicit measure the predictive confidence, serving as an implicit confidence measurement,
In contrast to several SSL algorithms, Tri-training does not require different supervised algorithms as base learners which leads to greater applicability in many real world classification problems [12, 46, 19]. A high-level description of Tri-training is presented in Algorithm 3.

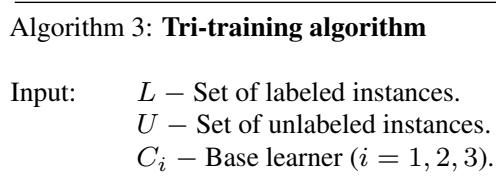

Output: Trained classifier.

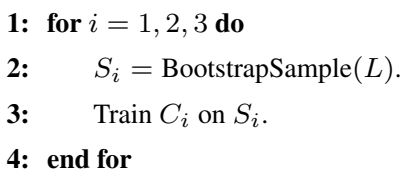

5: repeat

6: $\quad$ for $i=1,2,3$ do

7: $\quad L_{i}=\emptyset$.

8: $\quad$ for $u \in U$ do

9:

10

11:

12:

13:

14:

15:

17: end for

18: until some stopping criterion is met or $U$ is empty.

\section{An ensemble semi-supervised self-labeled algorithm}

In this section, the proposed ensemble SSL algorithm is presented which is based on the hybridization of ensemble learning with semi-supervised learning. Generally, the development of an ensemble of classifiers consists of two main steps: selection and combination.

The selection of the appropriate component classifiers which constitute the ensemble is considered essential for its efficiency and the key points for its efficacy is based on the diversity and the accuracy the component classifiers. A commonly and widely utilized approach is to apply diverse classification algorithms (with heterogeneous model representations) to a single dataset [24]. Moreover, the combination of the individual predictions of the classification algorithms takes place through several methodologies and techniques with different philosophy and performance $[28,9]$. 
By taking these into consideration, the development of an ensemble of classifiers is considered to be constituted by the SSL algorithms: Self-training, Co-training and Tri-training. These algorithms are self-labeled algorithms which exploit the hidden information in unlabeled data with complete different methodologies since Self-training and Tri-training are single-view methods while Co-training is a multi-view method.

A high-level description of the proposed Ensemble Semi-supervised Self-labeled Learning (EnSSL) algorithm is presented in Algorithm 4 which consists of two phases: Training phase and Testing phase.

In the Training phase, the SSL algorithms which constitute the ensemble are trained independently, using the same labeled $L$ and unlabeled $U$ datasets (steps 1-3). Clearly, the total computation time of this phase is the sum of computation times associated with each component SSL algorithm. In the Testing phase, initially the trained SSL algorithms are applied on each instance in the testing set (step 6). Subsequently, the individual predictions of the three SSL algorithms are combined via a maximum probability-based voting scheme. More specifically, the SSL algorithm which exhibits the most confident prediction over an unlabeled example of the test set is selected (step 8). In case the confidence of the prediction of the selected classifier meets a predefined threshold (ThresLev) then the classifier labels the example otherwise the prediction is not considered reliable enough (step 9). In this case, the output of the ensemble is defined as the combined predictions of three SSL learning algorithms via a simple majority voting, namely the ensemble output is the one made by more than half of them (step 11). This strategy has the advantage of exploiting the diversity of the errors of the learned models by using different classifiers and it does not require training on large quantities of representative recognition results from the individual learning algorithms.

\footnotetext{
Algorithm 4: EnSSL

Input: $\quad L-$ Set of labeled training instances.

$U-$ Set of unlabeled training instances.

$T$ - Set of test instances.

ThresLev - Threshold level.

Output: The labels of instances in the testing set.

I* Phase I: Training phase */

1: Train Self-train $(L, U)$.

2: Train Co-train $(L, U)$.

3: Train Tri-train $(L, U)$

/* Phase II: Testing phase */

5: for each $x$ from $T$ do

6: $\quad$ Apply Self-train, Co-train, Tri-train classifiers on $x$.
}

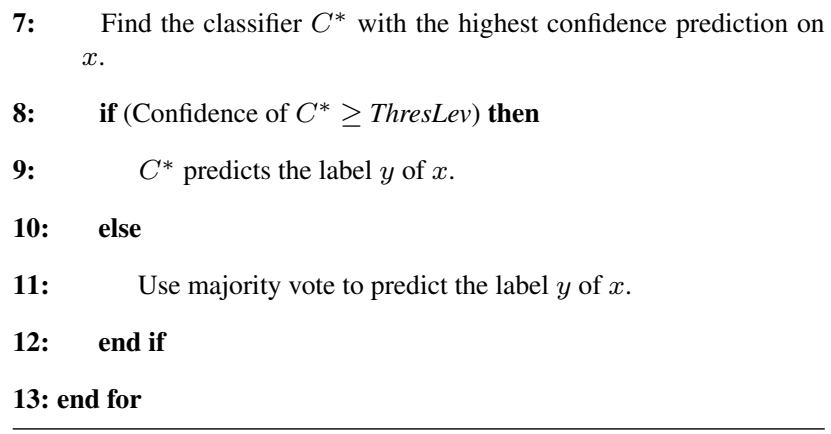

\section{Experimental results}

In this section, the classification performance of the proposed algorithm is compared with that of Self-training, Cotraining and Tri-training on 40 benchmark datasets from KEEL repository [2] in terms of classification accuracy.

Each self-labeled algorithm was evaluated deploying as base learners:

- C4.5 decision tree algorithm [26].

- RIPPER (JRip) [5] as the representative of the classification rules.

- $k \mathrm{NN}$ algorithm [1] as instance-based learner.

These algorithms probably constitute three of the most effective and most popular data mining algorithms for classification problems [39]. In order to study the influence of the amount of labeled data, four different ratios of the training data were used: $10 \%, 20 \%, 30 \%$ and $40 \%$. Moreover, we compared the classification performance of the proposed algorithm for each utilized base learner against the corresponding supervised learner.

The implementation code was written in JAVA, using WEKA Machine Learning Toolkit [13]. The configuration parameters of all the SSL methods and base learners used in the experiments are presented in Tables 1 and 2, respectively. It is worth noticing that the base learners were utilized with their the default parameter settings included in the WEKA software in order to minimize the effect of any expert bias by not attempting to tune any of the algorithms to the specific datasets.

Table 3 presents a brief description of the datasets structure i.e. number of instances (\#Instances), number of attributes (\#Features) and number of output classes (\#Classes). The datasets considered contain between 101 and 7400 instances, the number of attributes ranges from 3 to 90 and the number of classes varies between 2 and 15 . 


\begin{tabular}{ll}
\hline SSL Algorithm & Parameters \\
\hline Self-training & $\begin{array}{l}\text { Maximum number of iterations }=40 . \\
c=95 \% .\end{array}$ \\
\hline Co-training & $\begin{array}{l}\text { Maximum number of iterations }=40 . \\
\text { Initial unlabeled pool }=75 .\end{array}$ \\
\hline Tri-training & No parameters specified. \\
\hline EnSSL & ThresLev $=95 \%$. \\
\hline
\end{tabular}

Table 1: Parameter specification for all SSL algorithms employed in the experimentation.

\begin{tabular}{ll}
\hline Base learner & Parameters \\
\hline C4.5 & Confidence factor used for pruning $=0.25$. \\
& Minimum number of instances per leaf $=2$. \\
& Number of folds used for reduced-error pruning $=$ \\
& 3. \\
& Pruning is performed after tree building. \\
& Number of optimization runs $=2$. \\
JRip & Number of folds used for reduced-error pruning $=$ \\
& 3. \\
& Minimum total weight of the instances in a rule $=$ \\
& 2.0. \\
& Pruning is performed after tree building. \\
\hline$k \mathrm{NN}$ & Number of neighbors $=3$. \\
& Euclidean distance. \\
\hline
\end{tabular}

Table 2: Parameter specification for all base learners employed in the experimentation.

\begin{tabular}{|c|c|c|c|}
\hline Dataset & \#Instances & \#Features & \#Classes \\
\hline automobile & 159 & 15 & 2 \\
\hline appendicitis & 106 & 7 & 2 \\
\hline australian & 690 & 14 & 2 \\
\hline automobile & 205 & 26 & 7 \\
\hline breast & 286 & 9 & 2 \\
\hline bupa & 345 & 6 & 2 \\
\hline chess & 3196 & 36 & 2 \\
\hline contraceptive & 1473 & 9 & 3 \\
\hline dermatology & 358 & 34 & 6 \\
\hline ecoli & 336 & 7 & 8 \\
\hline flare & 1066 & 9 & 2 \\
\hline glass & 214 & 9 & 7 \\
\hline haberman & 306 & 3 & 2 \\
\hline heart & 270 & 13 & 2 \\
\hline housevotes & 435 & 16 & 2 \\
\hline iris & 150 & 4 & 3 \\
\hline led7digit & 500 & 7 & 10 \\
\hline lymph & 148 & 18 & 4 \\
\hline mammographic & 961 & 5 & 2 \\
\hline movement & 360 & 90 & 15 \\
\hline page-blocks & 5472 & 10 & 5 \\
\hline phoneme & 5404 & 5 & 2 \\
\hline pima & 768 & 8 & 2 \\
\hline ring & 7400 & 20 & 2 \\
\hline satimage & 6435 & 36 & 7 \\
\hline segment & 2310 & 19 & 7 \\
\hline
\end{tabular}

\begin{tabular}{lrrr}
\hline (continued). & & & \\
\hline Dataset & \#Instances & \#Features & \#Classes \\
\hline sonar & 208 & 60 & 2 \\
spambase & 4597 & 57 & 2 \\
spectheart & 267 & 44 & 2 \\
texture & 5500 & 40 & 11 \\
thyroid & 7200 & 21 & 3 \\
tic-tac-toe & 958 & 9 & 2 \\
titanic & 2201 & 3 & 2 \\
twonorm & 7400 & 20 & 2 \\
vehicle & 846 & 18 & 4 \\
vowel & 990 & 13 & 11 \\
wisconsin & 683 & 9 & 2 \\
wine & 178 & 13 & 3 \\
yeast & 1484 & 8 & 10 \\
zoo & 101 & 17 & 7 \\
\hline
\end{tabular}

Table 3: Brief description of datasets.

Tables 4-7 present the experimental results using 10\%, $20 \%, 30 \%$ and $40 \%$ labeled ratio, respectively regarding all base learners.

Table 8 presents the number of wins of each one of the tested algorithms according to the supervised classifier used as base learner and utilized the ratio of labeled data in the training, while the best scores are highlighted in bold. It should be mentioned that draw cases between algorithms have not been encountered. Clearly, the presented results illustrated that EnSSL is the most effective method in all cases except the one using $k \mathrm{NN}$ as base learner with a labeled ratio of $30 \%$. In this case, Tri-training performs better in 13 datasets, followed by EnSSL (9 wins). It is worth noticing that

- Depending upon the the ratio of labeled instances in the training set, EnSSL illustrates the highest classification accuracy in $46.2 \%$ of the datasets for $10 \%$ labeled ratio, $40 \%$ of the datasets for labeled ratio $20 \%$, $44.4 \%$ of the datasets for labeled ratio $30 \%$ and $44.4 \%$ of the datasets for $40 \%$ labeled ratio. Obviously, EnSSL exhibits better classification accuracy for $10 \%$ and $40 \%$ labeled ratio.

- Regarding the base classifier, EnSSL (C4.5) presents the best classification accuracy in 14, 20, 21 and 19 of the datasets using a labeled ratio of $10 \%, 20 \%, 30 \%$ and $40 \%$, respectively. EnSSL (JRip) prevails in 18, 14,16 and 16 of the datasets using a labeled ratio of $10 \%, 20 \%, 30 \%$ and $40 \%$, respectively. EnSSL ( $k N N)$ exhibit the best performance in 11,9 , and 17 of the datasets using a labeled ratio of $10 \%, 20 \%, 30 \%$ and $40 \%$, respectively. Hence, EnSSL performs better using C4.5 and JRip as base learners. 


\begin{tabular}{|c|c|c|c|c|c|c|c|c|c|c|c|c|c|c|c|}
\hline Dataset & $\mathrm{C} 4.5$ & $\begin{array}{c}\text { Self } \\
(\mathrm{C} 4.5)\end{array}$ & $\begin{array}{c}\text { Co } \\
\text { (C4.5) }\end{array}$ & $\begin{array}{c}\text { Tri } \\
\text { (C4.5) }\end{array}$ & $\begin{array}{l}\text { EnSSL } \\
\text { (C4.5) }\end{array}$ & JRip & $\begin{array}{c}\text { Self } \\
\text { (JRip) }\end{array}$ & $\begin{array}{c}\text { Co } \\
\text { (JRip) }\end{array}$ & $\begin{array}{c}\text { Tri } \\
\text { (JRip) }\end{array}$ & $\begin{array}{c}\text { EnSSL } \\
\text { (JRip) }\end{array}$ & $k \mathrm{NN}$ & $\begin{array}{c}\text { Self } \\
(k \mathrm{NN})\end{array}$ & $\begin{array}{c}\mathrm{Co} \\
(k \mathrm{NN})\end{array}$ & $\begin{array}{c}\text { Tri } \\
(k \mathrm{NN})\end{array}$ & $\begin{array}{l}\text { EnSSL } \\
(k \mathrm{NN})\end{array}$ \\
\hline automobile & $64,21 \%$ & $71,63 \%$ & $71,58 \%$ & $66,46 \%$ & $69,79 \%$ & $64,88 \%$ & $69,08 \%$ & $70,33 \%$ & $64,63 \%$ & $65,33 \%$ & $61,75 \%$ & $72,29 \%$ & $64,13 \%$ & $69,00 \%$ & $74,13 \%$ \\
\hline appendicitis & $76,27 \%$ & $81,09 \%$ & $83,00 \%$ & $82,00 \%$ & $82,00 \%$ & $83,91 \%$ & $82,09 \%$ & $81,00 \%$ & $83,09 \%$ & $83,09 \%$ & $82,00 \%$ & $85,82 \%$ & $85,82 \%$ & $85,82 \%$ & $85,82 \%$ \\
\hline australian & $84,20 \%$ & $85,80 \%$ & $85,65 \%$ & $87,10 \%$ & $86,67 \%$ & $85,22 \%$ & $85,65 \%$ & $85,36 \%$ & $86,23 \%$ & $86,38 \%$ & $83,19 \%$ & $83,91 \%$ & $85,36 \%$ & $83,77 \%$ & $84,93 \%$ \\
\hline banana & $74,40 \%$ & $74,58 \%$ & $74,85 \%$ & $75,00 \%$ & $74,85 \%$ & $73,19 \%$ & $72,89 \%$ & $73,15 \%$ & $73,25 \%$ & $73,30 \%$ & $72,38 \%$ & $72,89 \%$ & $73,15 \%$ & $73,25 \%$ & $73,30 \%$ \\
\hline breast & $70,22 \%$ & $75,87 \%$ & $75,54 \%$ & $73,82 \%$ & $75,54 \%$ & $68,45 \%$ & $69,91 \%$ & $67,81 \%$ & $73,12 \%$ & $69,56 \%$ & $73,03 \%$ & $72,41 \%$ & $73,09 \%$ & $73,45 \%$ & $73,45 \%$ \\
\hline bupa & $56,24 \%$ & $57,98 \%$ & $57,96 \%$ & $57,96 \%$ & $58,57 \%$ & $56,24 \%$ & $58,57 \%$ & $57,96 \%$ & $57,96 \%$ & $57,96 \%$ & $56,24 \%$ & $58,57 \%$ & $57,96 \%$ & $57,96 \%$ & $57,96 \%$ \\
\hline chess & $98,97 \%$ & $99,41 \%$ & $97,62 \%$ & $99,44 \%$ & $99,41 \%$ & $97,97 \%$ & $99,09 \%$ & $97,68 \%$ & $99,09 \%$ & $99,19 \%$ & $93,90 \%$ & $96,34 \%$ & $90,02 \%$ & $96,56 \%$ & $96,40 \%$ \\
\hline contraceptive & $48,75 \%$ & $49,69 \%$ & $50,98 \%$ & $50,37 \%$ & $50,30 \%$ & $43,04 \%$ & $43,65 \%$ & $46,64 \%$ & $46,57 \%$ & $46,77 \%$ & $48,95 \%$ & $50,84 \%$ & $51,12 \%$ & $51,59 \%$ & $51,12 \%$ \\
\hline dermatology & $92,60 \%$ & $94,54 \%$ & $90,17 \%$ & $94,54 \%$ & $95,36 \%$ & $85,76 \%$ & $87,15 \%$ & $86,06 \%$ & $89,61 \%$ & $91,00 \%$ & $94,79 \%$ & $97,25 \%$ & $94,53 \%$ & $97,24 \%$ & $96,97 \%$ \\
\hline ecoli & $79,77 \%$ & $80,37 \%$ & $74,99 \%$ & $80,97 \%$ & $79,78 \%$ & $78,83 \%$ & $77,99 \%$ & $75,88 \%$ & $79,48 \%$ & $78,88 \%$ & $80,93 \%$ & $80,97 \%$ & $77,37 \%$ & $82,15 \%$ & $82,15 \%$ \\
\hline flare & $72,23 \%$ & $74,66 \%$ & $71,76 \%$ & $73,73 \%$ & $74,10 \%$ & $68,38 \%$ & $71,20 \%$ & $67,18 \%$ & $70,44 \%$ & $70,36 \%$ & $72,04 \%$ & $74,95 \%$ & $63,32 \%$ & $73,92 \%$ & $74,20 \%$ \\
\hline glass & $63,51 \%$ & $67,81 \%$ & $62,73 \%$ & $64,48 \%$ & $67,32 \%$ & $61,21 \%$ & $68,25 \%$ & $62,64 \%$ & $55,30 \%$ & $64,09 \%$ & $64,03 \%$ & $72,51 \%$ & $71,56 \%$ & $72,97 \%$ & $73,44 \%$ \\
\hline haberman & $71,90 \%$ & $72,24 \%$ & $70,24 \%$ & $70,24 \%$ & $70,24 \%$ & $70,91 \%$ & $71,57 \%$ & $70,26 \%$ & $70,56 \%$ & $70,90 \%$ & $71,55 \%$ & $70,89 \%$ & $73,88 \%$ & $74,20 \%$ & $74,20 \%$ \\
\hline heart & $78,54 \%$ & $78,57 \%$ & $76,89 \%$ & $80,53 \%$ & $81,52 \%$ & $78,92 \%$ & $80,89 \%$ & $80,23 \%$ & $80,90 \%$ & $81,23 \%$ & $80,87 \%$ & $79,88 \%$ & $80,86 \%$ & $81,19 \%$ & $80,20 \%$ \\
\hline housevotes & $96,52 \%$ & $96,56 \%$ & $94,84 \%$ & $93,51 \%$ & $95,69 \%$ & $96,96 \%$ & $96,56 \%$ & $96,58 \%$ & $93,51 \%$ & $95,69 \%$ & $91,34 \%$ & $91,85 \%$ & $91,85 \%$ & $91,85 \%$ & $91,85 \%$ \\
\hline iris & $92,67 \%$ & $94,00 \%$ & $95,33 \%$ & $94,67 \%$ & $94,00 \%$ & $92,00 \%$ & $93,33 \%$ & $91,33 \%$ & $90,00 \%$ & $94,00 \%$ & $92,67 \%$ & $93,33 \%$ & $93,33 \%$ & $95,33 \%$ & $94,67 \%$ \\
\hline led7digit & $69,80 \%$ & $71,80 \%$ & $58,60 \%$ & $53,20 \%$ & $69,40 \%$ & $68,00 \%$ & $70,60 \%$ & $69,00 \%$ & $34,20 \%$ & $69,80 \%$ & $72,60 \%$ & $73,00 \%$ & $56,00 \%$ & $53,00 \%$ & $69,40 \%$ \\
\hline lymph & $70,95 \%$ & $74,38 \%$ & $73,76 \%$ & $73,71 \%$ & $73,71 \%$ & $72,90 \%$ & $74,29 \%$ & $75,05 \%$ & $72,29 \%$ & $74,38 \%$ & $76,95 \%$ & $78,48 \%$ & $80,57 \%$ & $81,19 \%$ & $80,48 \%$ \\
\hline mammographic & $82,41 \%$ & $83,49 \%$ & $83,01 \%$ & $84,22 \%$ & $84,34 \%$ & $82,41 \%$ & $83,25 \%$ & $82,29 \%$ & $83,86 \%$ & $83,73 \%$ & $82,05 \%$ & $82,65 \%$ & $82,29 \%$ & $83,73 \%$ & $83,25 \%$ \\
\hline movement & $40,28 \%$ & $56,94 \%$ & $50,00 \%$ & $35,83 \%$ & $52,78 \%$ & $29,44 \%$ & $56,94 \%$ & $49,17 \%$ & $31,94 \%$ & $48,89 \%$ & $40,28 \%$ & $65,00 \%$ & $56,94 \%$ & $59,72 \%$ & $65,56 \%$ \\
\hline page-blocks & $95,39 \%$ & $96,58 \%$ & $95,71 \%$ & $96,49 \%$ & $96,71 \%$ & $95,96 \%$ & $96,09 \%$ & $95,65 \%$ & $96,36 \%$ & $96,47 \%$ & $96,05 \%$ & $96,27 \%$ & $95,34 \%$ & $96,27 \%$ & $96,16 \%$ \\
\hline phoneme & $80,33 \%$ & $81,79 \%$ & $80,13 \%$ & $81,24 \%$ & $81,98 \%$ & $79,40 \%$ & $81,35 \%$ & $80,16 \%$ & $80,46 \%$ & $81,46 \%$ & $80,26 \%$ & $82,27 \%$ & $81,25 \%$ & $81,87 \%$ & $82,14 \%$ \\
\hline pima & $74,47 \%$ & $73,81 \%$ & $73,81 \%$ & $74,46 \%$ & $74,20 \%$ & $74,47 \%$ & $73,29 \%$ & $72,90 \%$ & $73,81 \%$ & $73,16 \%$ & $72,69 \%$ & $72,38 \%$ & $73,03 \%$ & $73,15 \%$ & $73,54 \%$ \\
\hline ring & $80,41 \%$ & $80,82 \%$ & $80,91 \%$ & $81,20 \%$ & $83,54 \%$ & $91,84 \%$ & $92,47 \%$ & $92,62 \%$ & $92,61 \%$ & $93,08 \%$ & $62,15 \%$ & $61,66 \%$ & $60,51 \%$ & $62,19 \%$ & $61,05 \%$ \\
\hline satimage & $83,20 \%$ & $84,38 \%$ & $83,98 \%$ & $84,65 \%$ & $85,39 \%$ & $83,31 \%$ & $83,62 \%$ & $84,15 \%$ & $83,43 \%$ & $84,80 \%$ & $88,48 \%$ & $89,25 \%$ & $88,47 \%$ & $89,03 \%$ & $89,46 \%$ \\
\hline segment & $92,55 \%$ & $94,42 \%$ & $90,30 \%$ & $93,90 \%$ & $94,89 \%$ & $91,82 \%$ & $90,87 \%$ & $86,15 \%$ & $90,09 \%$ & $92,77 \%$ & $93,33 \%$ & $93,12 \%$ & $90,52 \%$ & $93,29 \%$ & $93,77 \%$ \\
\hline sonar & $67,43 \%$ & $73,57 \%$ & $68,67 \%$ & $71,19 \%$ & $71,19 \%$ & $68,86 \%$ & $77,05 \%$ & $72,69 \%$ & $74,71 \%$ & $76,12 \%$ & $70,69 \%$ & $78,95 \%$ & $74,10 \%$ & $73,67 \%$ & $76,05 \%$ \\
\hline spambase & $91,55 \%$ & $92,72 \%$ & $91,13 \%$ & $92,79 \%$ & $92,89 \%$ & $90,68 \%$ & $92,37 \%$ & $91,55 \%$ & $91,89 \%$ & $92,83 \%$ & $92,39 \%$ & $93,02 \%$ & $92,33 \%$ & $93,22 \%$ & $93,31 \%$ \\
\hline spectheart & $67,50 \%$ & $68,75 \%$ & $70,00 \%$ & $70,00 \%$ & $70,00 \%$ & $63,75 \%$ & $72,50 \%$ & $70,00 \%$ & $71,25 \%$ & $71,25 \%$ & $63,75 \%$ & $66,25 \%$ & $68,75 \%$ & $68,75 \%$ & $68,75 \%$ \\
\hline texture & $84,55 \%$ & $87,87 \%$ & $86,02 \%$ & $86,65 \%$ & $88,95 \%$ & $84,73 \%$ & $86,91 \%$ & $86,33 \%$ & $86,20 \%$ & $89,64 \%$ & $94,75 \%$ & $96,07 \%$ & $95,13 \%$ & $95,78 \%$ & $96,22 \%$ \\
\hline thyroid & $99,17 \%$ & $99,32 \%$ & $98,72 \%$ & $99,24 \%$ & $99,28 \%$ & $98,89 \%$ & $99,17 \%$ & $98,42 \%$ & $99,17 \%$ & $99,24 \%$ & $98,43 \%$ & $98,76 \%$ & $98,53 \%$ & $98,69 \%$ & $98,87 \%$ \\
\hline tic-tac-toe & $81,73 \%$ & $83,60 \%$ & $85,70 \%$ & $85,27 \%$ & $85,38 \%$ & $97,08 \%$ & $97,49 \%$ & $97,91 \%$ & $97,60 \%$ & $97,49 \%$ & $97,29 \%$ & $99,06 \%$ & $98,75 \%$ & $98,64 \%$ & $98,96 \%$ \\
\hline titanic & $77,15 \%$ & $76,83 \%$ & $77,60 \%$ & $77,65 \%$ & $77,82 \%$ & $77,06 \%$ & $77,19 \%$ & $76,92 \%$ & $77,65 \%$ & $77,69 \%$ & $77,06 \%$ & $76,83 \%$ & $77,69 \%$ & $77,60 \%$ & $77,65 \%$ \\
\hline twonorm & $78,99 \%$ & $79,54 \%$ & $79,50 \%$ & $79,51 \%$ & $82,19 \%$ & $83,99 \%$ & $84,82 \%$ & $84,39 \%$ & $84,19 \%$ & $86,61 \%$ & $93,39 \%$ & $93,59 \%$ & $93,69 \%$ & $93,70 \%$ & $94,61 \%$ \\
\hline vehicle & $66,55 \%$ & $70,33 \%$ & $66,78 \%$ & $68,66 \%$ & $70,44 \%$ & $62,17 \%$ & $60,87 \%$ & $60,04 \%$ & $61,34 \%$ & $60,99 \%$ & $64,90 \%$ & $70,69 \%$ & $67,97 \%$ & $69,38 \%$ & $70,33 \%$ \\
\hline vowel & $97,27 \%$ & $98,28 \%$ & $97,57 \%$ & $98,28 \%$ & $98,28 \%$ & $96,96 \%$ & $98,18 \%$ & $97,17 \%$ & $98,28 \%$ & $98,28 \%$ & $95,85 \%$ & $97,57 \%$ & $95,85 \%$ & $97,47 \%$ & $97,57 \%$ \\
\hline wisconsin & $94,57 \%$ & $94,56 \%$ & $93,57 \%$ & $94,13 \%$ & $94,56 \%$ & $93,99 \%$ & $95,85 \%$ & $93,84 \%$ & $94,98 \%$ & $95,12 \%$ & $96,42 \%$ & $96,70 \%$ & $96,28 \%$ & $96,70 \%$ & $96,70 \%$ \\
\hline wine & $84,28 \%$ & $89,90 \%$ & $78,01 \%$ & $88,79 \%$ & $89,90 \%$ & $86,44 \%$ & $89,28 \%$ & $86,41 \%$ & $89,87 \%$ & $90,98 \%$ & $93,20 \%$ & $95,52 \%$ & $94,97 \%$ & $95,52 \%$ & $95,52 \%$ \\
\hline yeast & $75,13 \%$ & $74,93 \%$ & $74,86 \%$ & $74,86 \%$ & $74,86 \%$ & $75,07 \%$ & $74,19 \%$ & $75,74 \%$ & $75,13 \%$ & $75,20 \%$ & $75,21 \%$ & $74,19 \%$ & $75,07 \%$ & $75,27 \%$ & $75,14 \%$ \\
\hline zoo & $93,09 \%$ & $92,09 \%$ & $89,18 \%$ & $92,09 \%$ & $92,09 \%$ & $84,09 \%$ & $86,09 \%$ & $87,09 \%$ & $86,09 \%$ & $86,09 \%$ & $90,09 \%$ & $95,09 \%$ & $84,27 \%$ & $95,09 \%$ & $95,09 \%$ \\
\hline
\end{tabular}

Table 4: Classification accuracy (labeled ratio 10\%). 


\begin{tabular}{|c|c|c|c|c|c|c|c|c|c|c|c|c|c|c|c|}
\hline Dataset & $\mathrm{C} 4.5$ & $\begin{array}{c}\text { Self } \\
(\mathrm{C} 4.5)\end{array}$ & $\begin{array}{c}\text { Co } \\
\text { (C4.5) }\end{array}$ & $\begin{array}{c}\text { Tri } \\
\text { (C4.5) }\end{array}$ & $\begin{array}{l}\text { EnSSL } \\
\text { (C4.5) }\end{array}$ & JRip & $\begin{array}{c}\text { Self } \\
\text { (JRip) }\end{array}$ & $\begin{array}{c}\text { Co } \\
\text { (JRip) }\end{array}$ & $\begin{array}{c}\text { Tri } \\
\text { (JRip) }\end{array}$ & $\begin{array}{c}\text { EnSSL } \\
\text { (JRip) }\end{array}$ & $k \mathrm{NN}$ & $\begin{array}{c}\text { Self } \\
(k \mathrm{NN})\end{array}$ & $\begin{array}{c}\mathrm{Co} \\
(k \mathrm{NN})\end{array}$ & $\begin{array}{c}\text { Tri } \\
(k \mathrm{NN})\end{array}$ & $\begin{array}{l}\text { EnSSL } \\
(k \mathrm{NN})\end{array}$ \\
\hline automobile & $66,08 \%$ & $77,29 \%$ & $62,75 \%$ & $73,50 \%$ & $76,00 \%$ & $65,42 \%$ & $69,67 \%$ & $64,67 \%$ & $71,50 \%$ & $74,04 \%$ & $64,17 \%$ & $68,46 \%$ & $65,92 \%$ & $72,25 \%$ & $74,08 \%$ \\
\hline appendicitis & $80,09 \%$ & $81,09 \%$ & $83,00 \%$ & $82,91 \%$ & $82,91 \%$ & $83,91 \%$ & $82,09 \%$ & $82,00 \%$ & $82,91 \%$ & $82,00 \%$ & $83,09 \%$ & $86,82 \%$ & $86,73 \%$ & $85,82 \%$ & $85,82 \%$ \\
\hline australian & $86,09 \%$ & $86,67 \%$ & $86,23 \%$ & $87,10 \%$ & $87,68 \%$ & $85,51 \%$ & $86,09 \%$ & $85,80 \%$ & $86,23 \%$ & $86,09 \%$ & $84,93 \%$ & $85,94 \%$ & $83,04 \%$ & $84,06 \%$ & $85,07 \%$ \\
\hline banana & $74,62 \%$ & $74,57 \%$ & $75,23 \%$ & $75,08 \%$ & $78,26 \%$ & $73,36 \%$ & $72,75 \%$ & $74,21 \%$ & $73,79 \%$ & $75,13 \%$ & $74,55 \%$ & $72,75 \%$ & $74,21 \%$ & $73,79 \%$ & $75,13 \%$ \\
\hline breast & $70,23 \%$ & $74,16 \%$ & $71,31 \%$ & $75,54 \%$ & $75,64 \%$ & $69,24 \%$ & $72,07 \%$ & $68,51 \%$ & $71,70 \%$ & $71,01 \%$ & $73,12 \%$ & $70,68 \%$ & $71,69 \%$ & $72,75 \%$ & $72,75 \%$ \\
\hline bupa & $57,41 \%$ & $58,27 \%$ & $57,96 \%$ & $57,96 \%$ & $58,57 \%$ & $57,10 \%$ & $58,27 \%$ & $57,96 \%$ & $57,96 \%$ & $57,96 \%$ & $57,10 \%$ & $57,41 \%$ & $57,96 \%$ & $57,96 \%$ & $57,96 \%$ \\
\hline chess & $99,00 \%$ & $99,41 \%$ & $98,18 \%$ & $99,37 \%$ & $99,41 \%$ & $98,87 \%$ & $99,09 \%$ & $98,15 \%$ & $99,03 \%$ & $99,06 \%$ & $94,90 \%$ & $95,99 \%$ & $91,02 \%$ & $96,71 \%$ & $96,40 \%$ \\
\hline contraceptive & $50,44 \%$ & $50,17 \%$ & $50,84 \%$ & $50,44 \%$ & $50,71 \%$ & $43,04 \%$ & $42,57 \%$ & $46,64 \%$ & $46,36 \%$ & $45,75 \%$ & $50,51 \%$ & $50,37 \%$ & $51,93 \%$ & $49,83 \%$ & $50,71 \%$ \\
\hline dermatology & $93,41 \%$ & $92,63 \%$ & $89,32 \%$ & $93,99 \%$ & $94,81 \%$ & $85,77 \%$ & $88,52 \%$ & $85,49 \%$ & $89,05 \%$ & $91,52 \%$ & $94,79 \%$ & $96,97 \%$ & $95,32 \%$ & $96,97 \%$ & $97,24 \%$ \\
\hline ecoli & $80,02 \%$ & $79,48 \%$ & $76,79 \%$ & $79,19 \%$ & $80,06 \%$ & $80,62 \%$ & $78,89 \%$ & $77,66 \%$ & $78,01 \%$ & $78,58 \%$ & $80,94 \%$ & $79,20 \%$ & $80,07 \%$ & $81,29 \%$ & $81,58 \%$ \\
\hline flare & $73,17 \%$ & $75,42 \%$ & $72,70 \%$ & $73,35 \%$ & $74,29 \%$ & $68,95 \%$ & $73,17 \%$ & $72,70 \%$ & $71,85 \%$ & $73,73 \%$ & $72,51 \%$ & $74,29 \%$ & $68,48 \%$ & $73,36 \%$ & $73,45 \%$ \\
\hline glass & $65,52 \%$ & $67,34 \%$ & $63,70 \%$ & $64,96 \%$ & $70,24 \%$ & $63,12 \%$ & $64,94 \%$ & $65,02 \%$ & $62,21 \%$ & $66,47 \%$ & $67,81 \%$ & $66,84 \%$ & $71,58 \%$ & $69,13 \%$ & $72,97 \%$ \\
\hline haberman & $72,24 \%$ & $70,24 \%$ & $70,24 \%$ & $70,24 \%$ & $70,24 \%$ & $71,27 \%$ & $70,24 \%$ & $70,27 \%$ & $69,91 \%$ & $70,24 \%$ & $71,87 \%$ & $70,59 \%$ & $73,56 \%$ & $73,56 \%$ & $73,24 \%$ \\
\hline heart & $79,25 \%$ & $77,89 \%$ & $77,60 \%$ & $79,22 \%$ & $80,20 \%$ & $80,88 \%$ & $78,58 \%$ & $76,89 \%$ & $79,56 \%$ & $79,57 \%$ & $80,92 \%$ & $81,53 \%$ & $82,86 \%$ & $80,86 \%$ & $81,52 \%$ \\
\hline housevotes & $96,52 \%$ & $96,56 \%$ & $95,69 \%$ & $93,51 \%$ & $95,69 \%$ & $96,96 \%$ & $96,99 \%$ & $96,99 \%$ & $93,08 \%$ & $94,38 \%$ & $91,79 \%$ & $91,85 \%$ & $91,85 \%$ & $91,85 \%$ & $91,85 \%$ \\
\hline iris & $94,00 \%$ & $94,00 \%$ & $93,33 \%$ & $93,33 \%$ & $93,33 \%$ & $93,33 \%$ & $93,33 \%$ & $91,33 \%$ & $93,33 \%$ & $93,33 \%$ & $93,33 \%$ & $93,33 \%$ & $94,00 \%$ & $93,33 \%$ & $94,67 \%$ \\
\hline led7digit & $70,40 \%$ & $71,00 \%$ & $65,60 \%$ & $68,00 \%$ & $70,20 \%$ & $69,60 \%$ & $70,00 \%$ & $70,80 \%$ & $58,80 \%$ & $70,40 \%$ & $73,00 \%$ & $73,80 \%$ & $67,00 \%$ & $69,40 \%$ & $71,20 \%$ \\
\hline lymph & $71,57 \%$ & $75,71 \%$ & $72,43 \%$ & $74,43 \%$ & $76,43 \%$ & $74,48 \%$ & $72,43 \%$ & $76,38 \%$ & $73,76 \%$ & $75,10 \%$ & $79,19 \%$ & $79,81 \%$ & $83,24 \%$ & $81,19 \%$ & $81,14 \%$ \\
\hline mammographic & $83,61 \%$ & $82,65 \%$ & $82,65 \%$ & $84,10 \%$ & $83,37 \%$ & $83,25 \%$ & $83,37 \%$ & $82,89 \%$ & $83,73 \%$ & $83,61 \%$ & $83,01 \%$ & $83,49 \%$ & $82,29 \%$ & $83,98 \%$ & $83,25 \%$ \\
\hline movement & $50,00 \%$ & $59,17 \%$ & $47,50 \%$ & $47,22 \%$ & $57,50 \%$ & $43,33 \%$ & $54,17 \%$ & $51,94 \%$ & $21,39 \%$ & $45,83 \%$ & $57,22 \%$ & $63,06 \%$ & $55,83 \%$ & $61,11 \%$ & $65,00 \%$ \\
\hline page-blocks & $96,36 \%$ & $96,75 \%$ & $96,02 \%$ & $96,58 \%$ & $96,78 \%$ & $96,22 \%$ & $96,49 \%$ & $95,74 \%$ & $96,55 \%$ & $96,71 \%$ & $96,13 \%$ & $96,40 \%$ & $95,69 \%$ & $96,18 \%$ & $96,16 \%$ \\
\hline phoneme & $80,51 \%$ & $81,33 \%$ & $80,00 \%$ & $81,20 \%$ & $81,79 \%$ & $79,94 \%$ & $81,12 \%$ & $80,11 \%$ & $81,05 \%$ & $81,55 \%$ & $81,25 \%$ & $82,12 \%$ & $81,49 \%$ & $81,81 \%$ & $82,35 \%$ \\
\hline pima & $74,48 \%$ & $74,33 \%$ & $73,15 \%$ & $73,29 \%$ & $73,81 \%$ & $74,62 \%$ & $74,73 \%$ & $73,41 \%$ & $73,28 \%$ & $73,67 \%$ & $73,47 \%$ & $74,07 \%$ & $73,54 \%$ & $73,68 \%$ & $73,67 \%$ \\
\hline ring & $81,00 \%$ & $80,69 \%$ & $81,12 \%$ & $80,91 \%$ & $83,76 \%$ & $92,28 \%$ & $92,62 \%$ & $92,16 \%$ & $93,01 \%$ & $93,14 \%$ & $62,20 \%$ & $61,36 \%$ & $60,58 \%$ & $62,38 \%$ & $61,04 \%$ \\
\hline satimage & $83,29 \%$ & $84,57 \%$ & $84,27 \%$ & $84,15 \%$ & $84,90 \%$ & $83,40 \%$ & $83,23 \%$ & $83,00 \%$ & $83,73 \%$ & $84,55 \%$ & $88,90 \%$ & $89,28 \%$ & $88,50 \%$ & $89,42 \%$ & $89,65 \%$ \\
\hline segment & $93,46 \%$ & $94,37 \%$ & $91,17 \%$ & $94,03 \%$ & $94,59 \%$ & $92,16 \%$ & $91,21 \%$ & $88,96 \%$ & $90,48 \%$ & $92,47 \%$ & $92,34 \%$ & $92,90 \%$ & $91,21 \%$ & $93,64 \%$ & $93,55 \%$ \\
\hline sonar & $70,76 \%$ & $71,24 \%$ & $73,12 \%$ & $73,62 \%$ & $76,07 \%$ & $70,71 \%$ & $69,81 \%$ & $75,07 \%$ & $70,26 \%$ & $69,83 \%$ & $74,50 \%$ & $75,98 \%$ & $74,64 \%$ & $78,86 \%$ & $79,88 \%$ \\
\hline spambase & $92,28 \%$ & $92,89 \%$ & $91,87 \%$ & $92,81 \%$ & $92,85 \%$ & $90,94 \%$ & $92,55 \%$ & $91,78 \%$ & $92,52 \%$ & $92,89 \%$ & $92,85 \%$ & $93,18 \%$ & $92,81 \%$ & $93,39 \%$ & $93,70 \%$ \\
\hline spectheart & $71,25 \%$ & $68,75 \%$ & $71,25 \%$ & $70,00 \%$ & $68,75 \%$ & $65,00 \%$ & $71,25 \%$ & $70,00 \%$ & $71,25 \%$ & $71,25 \%$ & $66,25 \%$ & $66,25 \%$ & $66,25 \%$ & $67,50 \%$ & $68,75 \%$ \\
\hline texture & $86,36 \%$ & $87,29 \%$ & $86,29 \%$ & $87,42 \%$ & $88,76 \%$ & $85,33 \%$ & $86,53 \%$ & $86,13 \%$ & $86,51 \%$ & $89,31 \%$ & $94,49 \%$ & $96,27 \%$ & $95,58 \%$ & $96,05 \%$ & $96,56 \%$ \\
\hline thyroid & $99,21 \%$ & $99,32 \%$ & $98,96 \%$ & $99,25 \%$ & $99,31 \%$ & $99,01 \%$ & $99,17 \%$ & $98,54 \%$ & $99,13 \%$ & $99,19 \%$ & $98,58 \%$ & $98,65 \%$ & $98,96 \%$ & $98,58 \%$ & $98,79 \%$ \\
\hline tic-tac-toe & $82,36 \%$ & $86,11 \%$ & $85,28 \%$ & $84,96 \%$ & $87,47 \%$ & $97,39 \%$ & $97,70 \%$ & $98,02 \%$ & $98,01 \%$ & $97,91 \%$ & $98,12 \%$ & $98,12 \%$ & $97,07 \%$ & $98,64 \%$ & $98,33 \%$ \\
\hline titanic & $77,19 \%$ & $77,06 \%$ & $77,19 \%$ & $77,65 \%$ & $77,24 \%$ & $77,15 \%$ & $77,46 \%$ & $75,69 \%$ & $77,65 \%$ & $77,65 \%$ & $77,15 \%$ & $76,92 \%$ & $77,06 \%$ & $77,33 \%$ & $76,96 \%$ \\
\hline twonorm & $79,74 \%$ & $79,58 \%$ & $79,39 \%$ & $79,64 \%$ & $82,70 \%$ & $84,11 \%$ & $83,72 \%$ & $84,16 \%$ & $84,07 \%$ & $86,62 \%$ & $93,50 \%$ & $93,73 \%$ & $93,61 \%$ & $93,73 \%$ & $94,69 \%$ \\
\hline vehicle & $68,56 \%$ & $71,26 \%$ & $66,78 \%$ & $70,09 \%$ & $71,62 \%$ & $62,54 \%$ & $60,17 \%$ & $59,92 \%$ & $61,11 \%$ & $60,63 \%$ & $65,37 \%$ & $67,50 \%$ & $67,73 \%$ & $70,21 \%$ & $69,97 \%$ \\
\hline vowel & $97,87 \%$ & $98,08 \%$ & $98,48 \%$ & $98,38 \%$ & $98,58 \%$ & $97,77 \%$ & $98,18 \%$ & $98,08 \%$ & $98,18 \%$ & $98,18 \%$ & $96,76 \%$ & $96,86 \%$ & $96,66 \%$ & $97,17 \%$ & $97,47 \%$ \\
\hline wisconsin & $94,70 \%$ & $94,28 \%$ & $94,57 \%$ & $94,13 \%$ & $94,42 \%$ & $94,42 \%$ & $95,71 \%$ & $95,56 \%$ & $95,99 \%$ & $95,70 \%$ & $96,42 \%$ & $96,85 \%$ & $96,56 \%$ & $96,85 \%$ & $96,70 \%$ \\
\hline wine & $88,82 \%$ & $89,90 \%$ & $87,61 \%$ & $85,42 \%$ & $87,68 \%$ & $89,90 \%$ & $88,76 \%$ & $84,15 \%$ & $89,93 \%$ & $89,90 \%$ & $93,24 \%$ & $95,52 \%$ & $94,41 \%$ & $95,52 \%$ & $95,52 \%$ \\
\hline yeast & $75,34 \%$ & $76,07 \%$ & $74,39 \%$ & $75,00 \%$ & $74,73 \%$ & $75,20 \%$ & $75,80 \%$ & $75,14 \%$ & $74,80 \%$ & $75,20 \%$ & $75,47 \%$ & $74,86 \%$ & $75,34 \%$ & $75,41 \%$ & $75,20 \%$ \\
\hline zoo & $94,00 \%$ & $92,09 \%$ & $82,18 \%$ & $89,09 \%$ & $91,09 \%$ & $86,09 \%$ & $84,18 \%$ & $89,00 \%$ & $86,09 \%$ & $86,09 \%$ & $92,09 \%$ & $95,09 \%$ & $81,27 \%$ & $94,18 \%$ & $94,18 \%$ \\
\hline
\end{tabular}

Table 5: Classification accuracy (labeled ratio 20\%). 


\begin{tabular}{|c|c|c|c|c|c|c|c|c|c|c|c|c|c|c|c|}
\hline Dataset & $\mathrm{C} 4.5$ & $\begin{array}{c}\text { Self } \\
(\mathrm{C} 4.5)\end{array}$ & $\begin{array}{c}\text { Co } \\
\text { (C4.5) }\end{array}$ & $\begin{array}{c}\text { Tri } \\
(\mathrm{C} 4.5)\end{array}$ & $\begin{array}{c}\text { EnSSL } \\
\text { (C4.5) }\end{array}$ & JRip & $\begin{array}{c}\text { Self } \\
\text { (JRip) }\end{array}$ & $\begin{array}{c}\text { Co } \\
\text { (JRip) }\end{array}$ & $\begin{array}{c}\text { Tri } \\
\text { (JRip) }\end{array}$ & $\begin{array}{c}\text { EnSSL } \\
\text { (JRip) }\end{array}$ & $k \mathrm{NN}$ & $\begin{array}{c}\text { Self } \\
(k \mathrm{NN})\end{array}$ & $\begin{array}{c}\mathrm{Co} \\
(k \mathrm{NN})\end{array}$ & $\begin{array}{c}\text { Tri } \\
(k \mathrm{NN})\end{array}$ & $\begin{array}{l}\text { EnSSL } \\
(k \mathrm{NN})\end{array}$ \\
\hline automobile & $21 \%$ & $73,46 \%$ & $72,92 \%$ & $77,29 \%$ & $79,21 \%$ & $67,92 \%$ & $63,42 \%$ & $70,38 \%$ & $71,54 \%$ & $72,83 \%$ & $65,50 \%$ & $61,63 \%$ & $69,17 \%$ & $70,96 \%$ & $70,33 \%$ \\
\hline appendicitis & $82,00 \%$ & $83,09 \%$ & $83,00 \%$ & $84,82 \%$ & $84,00 \%$ & $83,91 \%$ & $83,91 \%$ & $84,82 \%$ & $83,82 \%$ & $83,82 \%$ & $85,73 \%$ & $86,73 \%$ & $86,73 \%$ & $84,91 \%$ & $86,73 \%$ \\
\hline australian & $85,94 \%$ & $86,52 \%$ & $85,80 \%$ & $86,81 \%$ & $86,67 \%$ & $85,65 \%$ & $85,94 \%$ & $85,65 \%$ & $85,80 \%$ & $85,51 \%$ & $84,20 \%$ & $83,91 \%$ & $85,07 \%$ & $84,06 \%$ & $85,64 \%$ \\
\hline banana & $74,70 \%$ & $74,58 \%$ & $75,36 \%$ & $74,70 \%$ & $78,81 \%$ & $73,45 \%$ & $72,89 \%$ & $73,70 \%$ & $73,11 \%$ & $76,11 \%$ & $74,66 \%$ & $72,89 \%$ & $73,70 \%$ & $73,11 \%$ & $76,11 \%$ \\
\hline breast & $70,32 \%$ & $75,20 \%$ & $74,16 \%$ & $75,54 \%$ & $75,74 \%$ & $69,54 \%$ & $75,17 \%$ & $69,95 \%$ & $71,32 \%$ & $72,03 \%$ & $73,23 \%$ & $73,09 \%$ & $71,69 \%$ & $73,09 \%$ & $72,75 \%$ \\
\hline bupa & $57,10 \%$ & $57,98 \%$ & $57,96 \%$ & $57,96 \%$ & $58,57 \%$ & $57,41 \%$ & $57,98 \%$ & $55,67 \%$ & $57,96 \%$ & $57,96 \%$ & $57,41 \%$ & $55,92 \%$ & $57,96 \%$ & $57,96 \%$ & $57,96 \%$ \\
\hline chess & $99,12 \%$ & $99,41 \%$ & $98,28 \%$ & $99,41 \%$ & $99,44 \%$ & $98,90 \%$ & $99,00 \%$ & $98,12 \%$ & $99,22 \%$ & $99,31 \%$ & $94,96 \%$ & $94,15 \%$ & $92,49 \%$ & $96,71 \%$ & $95,93 \%$ \\
\hline contraceptive & $50,85 \%$ & $49,82 \%$ & $50,91 \%$ & $50,17 \%$ & $51,72 \%$ & $46,50 \%$ & $44,60 \%$ & $47,39 \%$ & $46,98 \%$ & $46,43 \%$ & $51,39 \%$ & $49,21 \%$ & $51,66 \%$ & $52,20 \%$ & $51,11 \%$ \\
\hline dermatology & $94,80 \%$ & $93,15 \%$ & $90,97 \%$ & $94,53 \%$ & $95,08 \%$ & $87,67 \%$ & $88,81 \%$ & $86,35 \%$ & $87,40 \%$ & $89,08 \%$ & $95,88 \%$ & $96,43 \%$ & $96,15 \%$ & $97,24 \%$ & $96,97 \%$ \\
\hline ecoli & $80,06 \%$ & $79,15 \%$ & $77,07 \%$ & $78,87 \%$ & $78,57 \%$ & $80,66 \%$ & $79,51 \%$ & $79,79 \%$ & $76,53 \%$ & $77,12 \%$ & $81,24 \%$ & $79,80 \%$ & $80,37 \%$ & $80,70 \%$ & $80,70 \%$ \\
\hline flare & $73,63 \%$ & $74,48 \%$ & $74,20 \%$ & $73,45 \%$ & $73,73 \%$ & $69,13 \%$ & $71,00 \%$ & $70,64 \%$ & $70,55 \%$ & $71,95 \%$ & $72,61 \%$ & $73,35 \%$ & $71,57 \%$ & $74,11 \%$ & $73,73 \%$ \\
\hline glass & $66,47 \%$ & $61,19 \%$ & $65,95 \%$ & $69,74 \%$ & $70,15 \%$ & $63,16 \%$ & $63,66 \%$ & $65,06 \%$ & $67,40 \%$ & $68,83 \%$ & $69,70 \%$ & $63,68 \%$ & $60,80 \%$ & $71,99 \%$ & $70,65 \%$ \\
\hline haberman & $72,24 \%$ & $71,86 \%$ & $70,24 \%$ & $70,24 \%$ & $70,24 \%$ & $71,91 \%$ & $71,86 \%$ & $70,90 \%$ & $70,24 \%$ & $70,24 \%$ & $72,89 \%$ & $70,91 \%$ & $72,57 \%$ & $73,54 \%$ & $72,56 \%$ \\
\hline heart & $79,90 \%$ & $76,27 \%$ & $79,87 \%$ & $78,88 \%$ & $80,22 \%$ & $81,23 \%$ & $79,59 \%$ & $79,22 \%$ & $82,22 \%$ & $81,87 \%$ & $82,22 \%$ & $80,19 \%$ & $83,84 \%$ & $81,52 \%$ & $81,84 \%$ \\
\hline housevotes & $96,52 \%$ & $96,56 \%$ & $96,99 \%$ & $96,56 \%$ & $96,56 \%$ & $96,96 \%$ & $96,99 \%$ & $96,56 \%$ & $96,99 \%$ & $96,99 \%$ & $92,21 \%$ & $91,85 \%$ & $91,85 \%$ & $92,26 \%$ & $91,85 \%$ \\
\hline iris & $94,00 \%$ & $94,00 \%$ & $94,00 \%$ & $93,33 \%$ & $94,00 \%$ & $93,33 \%$ & $93,33 \%$ & $92,00 \%$ & $94,00 \%$ & $93,33 \%$ & $93,33 \%$ & $94,00 \%$ & $94,00 \%$ & $92,00 \%$ & $93,33 \%$ \\
\hline led7digit & $71,20 \%$ & $70,40 \%$ & $69,20 \%$ & $71,00 \%$ & $71,00 \%$ & $70,40 \%$ & $69,20 \%$ & $71,60 \%$ & $69,00 \%$ & $71,00 \%$ & $73,20 \%$ & $73,60 \%$ & $70,80 \%$ & $70,80 \%$ & $71,80 \%$ \\
\hline lymph & $76,33 \%$ & $73,62 \%$ & $76,43 \%$ & $72,38 \%$ & $71,71 \%$ & $74,90 \%$ & $75,76 \%$ & $79,76 \%$ & $75,86 \%$ & $77,14 \%$ & $79,81 \%$ & $79,14 \%$ & $77,86 \%$ & $81,19 \%$ & $80,52 \%$ \\
\hline mammographic & $83,73 \%$ & $83,98 \%$ & $82,05 \%$ & $84,22 \%$ & $84,10 \%$ & $83,61 \%$ & $84,10 \%$ & $82,29 \%$ & $84,10 \%$ & $84,22 \%$ & $83,37 \%$ & $83,86 \%$ & $82,53 \%$ & $83,73 \%$ & $83,96 \%$ \\
\hline movement & $55,28 \%$ & $58,89 \%$ & $51,67 \%$ & $50,56 \%$ & $61,39 \%$ & $51,39 \%$ & $54,44 \%$ & $50,00 \%$ & $38,33 \%$ & $53,06 \%$ & $59,11 \%$ & $63,06 \%$ & $54,44 \%$ & $58,06 \%$ & $63,61 \%$ \\
\hline page-blocks & $96,38 \%$ & $96,47 \%$ & $96,38 \%$ & $96,69 \%$ & $96,87 \%$ & $96,29 \%$ & $96,36 \%$ & $96,11 \%$ & $96,38 \%$ & $96,60 \%$ & $96,20 \%$ & $96,20 \%$ & $95,92 \%$ & $96,33 \%$ & $96,34 \%$ \\
\hline phoneme & $81,05 \%$ & $81,01 \%$ & $80,11 \%$ & $81,31 \%$ & $81,42 \%$ & $80,61 \%$ & $80,55 \%$ & $80,64 \%$ & $80,88 \%$ & $81,44 \%$ & $81,68 \%$ & $81,98 \%$ & $81,35 \%$ & $82,20 \%$ & $82,14 \%$ \\
\hline pima & $75,53 \%$ & $74,84 \%$ & $73,68 \%$ & $74,72 \%$ & $75,24 \%$ & $75,25 \%$ & $73,80 \%$ & $72,65 \%$ & $72,37 \%$ & $73,02 \%$ & $74,48 \%$ & $74,51 \%$ & $74,20 \%$ & $72,76 \%$ & $74,71 \%$ \\
\hline ring & $81,23 \%$ & $80,30 \%$ & $81,43 \%$ & $81,03 \%$ & $83,15 \%$ & $92,59 \%$ & $92,88 \%$ & $91,80 \%$ & $92,59 \%$ & $92,88 \%$ & $62,36 \%$ & $61,15 \%$ & $60,65 \%$ & $62,26 \%$ & $60,80 \%$ \\
\hline satimage & $84,29 \%$ & $84,48 \%$ & $84,41 \%$ & $84,69 \%$ & $85,18 \%$ & $83,43 \%$ & $83,39 \%$ & $83,36 \%$ & $83,56 \%$ & $84,91 \%$ & $88,90 \%$ & $89,08 \%$ & $88,98 \%$ & $89,45 \%$ & $89,76 \%$ \\
\hline segment & $93,68 \%$ & $94,03 \%$ & $91,73 \%$ & $94,37 \%$ & $94,76 \%$ & $92,64 \%$ & $91,13 \%$ & $87,88 \%$ & $90,30 \%$ & $92,77 \%$ & $92,55 \%$ & $92,51 \%$ & $90,82 \%$ & $93,55 \%$ & $93,55 \%$ \\
\hline sonar & $72,62 \%$ & $71,69 \%$ & $74,57 \%$ & $76,10 \%$ & $74,17 \%$ & $74,55 \%$ & $74,14 \%$ & $71,69 \%$ & $74,10 \%$ & $76,50 \%$ & $74,52 \%$ & $77,50 \%$ & $76,43 \%$ & $72,21 \%$ & $74,10 \%$ \\
\hline spambase & $92,70 \%$ & $92,70 \%$ & $92,13 \%$ & $92,92 \%$ & $92,87 \%$ & $92,15 \%$ & $91,78 \%$ & $91,83 \%$ & $92,31 \%$ & $92,44 \%$ & $92,98 \%$ & $92,55 \%$ & $92,94 \%$ & $93,37 \%$ & $93,26 \%$ \\
\hline spectheart & $71,25 \%$ & $71,25 \%$ & $68,75 \%$ & $67,50 \%$ & $68,75 \%$ & $68,75 \%$ & $70,00 \%$ & $71,25 \%$ & $71,25 \%$ & $71,25 \%$ & $70,00 \%$ & $71,25 \%$ & $68,75 \%$ & $67,50 \%$ & $68,75 \%$ \\
\hline texture & $86,44 \%$ & $87,80 \%$ & $86,73 \%$ & $86,76 \%$ & $88,85 \%$ & $86,25 \%$ & $86,44 \%$ & $87,45 \%$ & $86,56 \%$ & $88,95 \%$ & $95,64 \%$ & $95,89 \%$ & $95,85 \%$ & $96,16 \%$ & $96,40 \%$ \\
\hline thyroid & $99,25 \%$ & $99,17 \%$ & $99,22 \%$ & $99,32 \%$ & $99,28 \%$ & $99,07 \%$ & $99,04 \%$ & $99,17 \%$ & $99,00 \%$ & $99,13 \%$ & $98,61 \%$ & $98,33 \%$ & $98,68 \%$ & $98,63 \%$ & $98,64 \%$ \\
\hline tic-tac-toe & $83,30 \%$ & $84,96 \%$ & $85,80 \%$ & $85,38 \%$ & $88,41 \%$ & $97,81 \%$ & $97,70 \%$ & $97,60 \%$ & $98,02 \%$ & $97,70 \%$ & $98,54 \%$ & $96,45 \%$ & $97,07 \%$ & $98,85 \%$ & $98,85 \%$ \\
\hline titanic & $77,15 \%$ & $77,28 \%$ & $77,46 \%$ & $77,10 \%$ & $77,24 \%$ & $77,24 \%$ & $77,24 \%$ & $77,46 \%$ & $77,51 \%$ & $77,24 \%$ & $77,17 \%$ & $77,19 \%$ & $77,46 \%$ & $77,19 \%$ & $77,06 \%$ \\
\hline twonorm & $79,85 \%$ & $79,53 \%$ & $79,68 \%$ & $81,18 \%$ & $83,59 \%$ & $84,82 \%$ & $83,93 \%$ & $84,73 \%$ & $84,91 \%$ & $87,38 \%$ & $93,72 \%$ & $93,88 \%$ & $93,73 \%$ & $93,93 \%$ & $94,89 \%$ \\
\hline vehicle & $68,68 \%$ & $70,45 \%$ & $69,15 \%$ & $69,74 \%$ & $71,75 \%$ & $62,77 \%$ & $58,52 \%$ & $60,64 \%$ & $60,76 \%$ & $60,76 \%$ & $67,73 \%$ & $66,20 \%$ & $67,86 \%$ & $70,21 \%$ & $69,04 \%$ \\
\hline vowel & $97,87 \%$ & $97,47 \%$ & $97,67 \%$ & $97,98 \%$ & $97,87 \%$ & $97,77 \%$ & $97,57 \%$ & $97,98 \%$ & $98,38 \%$ & $98,28 \%$ & $97,07 \%$ & $96,86 \%$ & $96,05 \%$ & $97,97 \%$ & $97,77 \%$ \\
\hline wisconsin & $94,99 \%$ & $94,99 \%$ & $94,13 \%$ & $94,42 \%$ & $94,85 \%$ & $95,28 \%$ & $96,42 \%$ & $94,41 \%$ & $94,99 \%$ & $94,98 \%$ & $96,57 \%$ & $96,70 \%$ & $96,56 \%$ & $96,70 \%$ & $96,70 \%$ \\
\hline wine & $89,35 \%$ & $88,79 \%$ & $87,61 \%$ & $91,57 \%$ & $91,57 \%$ & $91,57 \%$ & $87,58 \%$ & $88,73 \%$ & $88,79 \%$ & $88,17 \%$ & $94,35 \%$ & $96,08 \%$ & $96,63 \%$ & $95,52 \%$ & $96,08 \%$ \\
\hline yeast & $75,41 \%$ & $74,73 \%$ & $75,20 \%$ & $75,20 \%$ & $75,54 \%$ & $76,08 \%$ & $75,13 \%$ & $75,00 \%$ & $75,54 \%$ & $76,21 \%$ & $75,68 \%$ & $74,53 \%$ & $74,59 \%$ & $75,20 \%$ & $75,07 \%$ \\
\hline zoo & $94,00 \%$ & $93,09 \%$ & $88,09 \%$ & $94,00 \%$ & $95,00 \%$ & $87,09 \%$ & $87,09 \%$ & $81,18 \%$ & $86,09 \%$ & $86,09 \%$ & $93,01 \%$ & $94,09 \%$ & $88,27 \%$ & $93,09 \%$ & $93,09 \%$ \\
\hline
\end{tabular}

Table 6: Classification accuracy (labeled ratio 30\%). 


\begin{tabular}{|c|c|c|c|c|c|c|c|c|c|c|c|c|c|c|c|}
\hline Dataset & $\mathrm{C} 4.5$ & $\begin{array}{c}\text { Self } \\
(\mathrm{C} 4.5)\end{array}$ & $\begin{array}{c}\text { Co } \\
\text { (C4.5) }\end{array}$ & $\begin{array}{c}\text { Tri } \\
\text { (C4.5) }\end{array}$ & $\begin{array}{l}\text { EnSSL } \\
\text { (C4.5) }\end{array}$ & JRip & $\begin{array}{c}\text { Self } \\
\text { (JRip) }\end{array}$ & $\begin{array}{c}\text { Co } \\
\text { (JRip) }\end{array}$ & $\begin{array}{c}\text { Tri } \\
\text { (JRip) }\end{array}$ & $\begin{array}{c}\text { EnSSL } \\
\text { (JRip) }\end{array}$ & $k \mathrm{NN}$ & $\begin{array}{c}\text { Self } \\
(k \mathrm{NN})\end{array}$ & $\begin{array}{c}\mathrm{Co} \\
(k \mathrm{NN})\end{array}$ & $\begin{array}{c}\text { Tri } \\
(k \mathrm{NN})\end{array}$ & $\begin{array}{l}\text { EnSSL } \\
(k \mathrm{NN})\end{array}$ \\
\hline automobile & $74,25 \%$ & $72,33 \%$ & $77,33 \%$ & $75,46 \%$ & $81,13 \%$ & $70,88 \%$ & $59,71 \%$ & $68,46 \%$ & $70,96 \%$ & $71,58 \%$ & $67,92 \%$ & $65,33 \%$ & $64,75 \%$ & $67,21 \%$ & $69,75 \%$ \\
\hline appendicitis & $83,82 \%$ & $81,09 \%$ & $85,73 \%$ & $82,00 \%$ & $82,00 \%$ & $83,91 \%$ & $81,09 \%$ & $83,82 \%$ & $83,00 \%$ & $83,00 \%$ & $85,81 \%$ & $82,09 \%$ & $85,82 \%$ & $84,91 \%$ & $85,82 \%$ \\
\hline australian & $86,23 \%$ & $85,80 \%$ & $86,09 \%$ & $87,54 \%$ & $87,10 \%$ & $85,65 \%$ & $85,36 \%$ & $85,94 \%$ & $86,38 \%$ & $85,36 \%$ & $85,38 \%$ & $84,93 \%$ & $84,06 \%$ & $84,20 \%$ & $86,78 \%$ \\
\hline banana & $74,79 \%$ & $74,66 \%$ & $75,77 \%$ & $74,72 \%$ & $80,53 \%$ & $73,47 \%$ & $72,74 \%$ & $73,55 \%$ & $72,81 \%$ & $75,70 \%$ & $74,94 \%$ & $72,74 \%$ & $73,55 \%$ & $72,81 \%$ & $75,70 \%$ \\
\hline breast & $70,95 \%$ & $71,34 \%$ & $75,20 \%$ & $75,16 \%$ & $75,16 \%$ & $70,41 \%$ & $70,68 \%$ & $70,33 \%$ & $71,70 \%$ & $70,67 \%$ & $73,04 \%$ & $72,73 \%$ & $72,38 \%$ & $72,75 \%$ & $73,08 \%$ \\
\hline bupa & $58,04 \%$ & $54,75 \%$ & $57,67 \%$ & $57,96 \%$ & $58,57 \%$ & $57,44 \%$ & $54,75 \%$ & $57,67 \%$ & $55,67 \%$ & $57,96 \%$ & $57,54 \%$ & $55,34 \%$ & $57,67 \%$ & $57,96 \%$ & $57,96 \%$ \\
\hline chess & $99,22 \%$ & $99,25 \%$ & $99,03 \%$ & $99,41 \%$ & $99,41 \%$ & $99,00 \%$ & $99,19 \%$ & $98,62 \%$ & $99,12 \%$ & $99,16 \%$ & $95,71 \%$ & $93,55 \%$ & $93,30 \%$ & $96,65 \%$ & $95,96 \%$ \\
\hline contraceptive & $51,41 \%$ & $48,00 \%$ & $51,73 \%$ & $50,03 \%$ & $51,52 \%$ & $46,87 \%$ & $42,84 \%$ & $46,98 \%$ & $47,05 \%$ & $46,88 \%$ & $51,96 \%$ & $47,93 \%$ & $51,11 \%$ & $52,07 \%$ & $51,93 \%$ \\
\hline dermatology & $95,08 \%$ & $93,46 \%$ & $92,05 \%$ & $94,26 \%$ & $95,38 \%$ & $87,71 \%$ & $87,98 \%$ & $88,25 \%$ & $89,08 \%$ & $90,17 \%$ & $96,14 \%$ & $96,43 \%$ & $95,59 \%$ & $97,24 \%$ & $97,24 \%$ \\
\hline ecoli & $81,84 \%$ & $77,67 \%$ & $80,63 \%$ & $79,48 \%$ & $80,34 \%$ & $81,22 \%$ & $79,49 \%$ & $77,69 \%$ & $80,37 \%$ & $79,80 \%$ & $82,04 \%$ & $80,96 \%$ & $79,46 \%$ & $80,69 \%$ & $82,47 \%$ \\
\hline flare & $73,82 \%$ & $73,63 \%$ & $73,07 \%$ & $74,29 \%$ & $74,10 \%$ & $69,23 \%$ & $68,86 \%$ & $71,76 \%$ & $69,79 \%$ & $70,64 \%$ & $73,27 \%$ & $73,17 \%$ & $72,32 \%$ & $73,64 \%$ & $73,36 \%$ \\
\hline glass & $70,65 \%$ & $61,58 \%$ & $67,38 \%$ & $68,72 \%$ & $72,01 \%$ & $66,76 \%$ & $55,13 \%$ & $67,79 \%$ & $61,77 \%$ & $67,79 \%$ & $73,42 \%$ & $62,19 \%$ & $70,17 \%$ & $73,40 \%$ & $74,78 \%$ \\
\hline haberman & $73,53 \%$ & $73,53 \%$ & $71,90 \%$ & $70,24 \%$ & $70,24 \%$ & $72,20 \%$ & $72,86 \%$ & $70,94 \%$ & $69,27 \%$ & $69,27 \%$ & $72,91 \%$ & $72,22 \%$ & $73,87 \%$ & $74,20 \%$ & $74,20 \%$ \\
\hline heart & $80,23 \%$ & $74,94 \%$ & $77,95 \%$ & $77,90 \%$ & $80,88 \%$ & $81,55 \%$ & $80,26 \%$ & $82,47 \%$ & $82,22 \%$ & $83,52 \%$ & $82,87 \%$ & $81,53 \%$ & $82,52 \%$ & $80,86 \%$ & $82,49 \%$ \\
\hline housevotes & $96,56 \%$ & $94,82 \%$ & $96,12 \%$ & $96,56 \%$ & $96,56 \%$ & $96,96 \%$ & $96,99 \%$ & $96,56 \%$ & $96,56 \%$ & $96,56 \%$ & $92,23 \%$ & $91,85 \%$ & $92,26 \%$ & $91,85 \%$ & $91,85 \%$ \\
\hline iris & $94,00 \%$ & $94,00 \%$ & $93,33 \%$ & $93,33 \%$ & $93,33 \%$ & $94,00 \%$ & $94,00 \%$ & $86,67 \%$ & $93,33 \%$ & $93,33 \%$ & $94,00 \%$ & $94,00 \%$ & $94,00 \%$ & $92,67 \%$ & $93,33 \%$ \\
\hline led7digit & $71,40 \%$ & $68,60 \%$ & $68,40 \%$ & $70,40 \%$ & $70,80 \%$ & $70,80 \%$ & $69,60 \%$ & $68,80 \%$ & $70,80 \%$ & $71,00 \%$ & $73,40 \%$ & $74,00 \%$ & $72,00 \%$ & $71,80 \%$ & $72,20 \%$ \\
\hline lymph & $76,33 \%$ & $75,10 \%$ & $74,29 \%$ & $75,05 \%$ & $75,05 \%$ & $76,24 \%$ & $76,43 \%$ & $77,86 \%$ & $75,76 \%$ & $77,24 \%$ & $80,52 \%$ & $76,43 \%$ & $79,81 \%$ & $81,86 \%$ & $81,86 \%$ \\
\hline mammographic & $83,73 \%$ & $83,61 \%$ & $82,29 \%$ & $84,10 \%$ & $84,10 \%$ & $83,86 \%$ & $83,61 \%$ & $82,89 \%$ & $84,22 \%$ & $83,49 \%$ & $83,37 \%$ & $82,29 \%$ & $82,29 \%$ & $83,61 \%$ & $83,13 \%$ \\
\hline movement & $55,83 \%$ & $58,89 \%$ & $51,11 \%$ & $55,00 \%$ & $59,17 \%$ & $52,44 \%$ & $50,28 \%$ & $50,00 \%$ & $49,17 \%$ & $52,78 \%$ & $61,39 \%$ & $53,89 \%$ & $58,89 \%$ & $65,28 \%$ & $62,78 \%$ \\
\hline page-blocks & $96,42 \%$ & $96,56 \%$ & $96,36 \%$ & $96,77 \%$ & $96,91 \%$ & $96,34 \%$ & $96,34 \%$ & $96,29 \%$ & $96,24 \%$ & $96,34 \%$ & $96,31 \%$ & $96,27 \%$ & $96,05 \%$ & $96,31 \%$ & $96,40 \%$ \\
\hline phoneme & $81,11 \%$ & $80,51 \%$ & $80,66 \%$ & $81,20 \%$ & $81,25 \%$ & $80,90 \%$ & $80,05 \%$ & $80,48 \%$ & $81,03 \%$ & $81,18 \%$ & $82,14 \%$ & $81,61 \%$ & $81,53 \%$ & $82,11 \%$ & $82,20 \%$ \\
\hline pima & $74,87 \%$ & $73,54 \%$ & $74,33 \%$ & $73,16 \%$ & $74,20 \%$ & $76,05 \%$ & $73,80 \%$ & $73,81 \%$ & $73,16 \%$ & $74,33 \%$ & $74,57 \%$ & $74,19 \%$ & $74,34 \%$ & $73,02 \%$ & $74,84 \%$ \\
\hline ring & $82,45 \%$ & $80,91 \%$ & $80,97 \%$ & $81,16 \%$ & $83,32 \%$ & $92,69 \%$ & $92,96 \%$ & $91,64 \%$ & $92,74 \%$ & $93,19 \%$ & $62,72 \%$ & $60,47 \%$ & $60,47 \%$ & $62,32 \%$ & $60,49 \%$ \\
\hline satimage & $84,38 \%$ & $84,34 \%$ & $84,55 \%$ & $84,24 \%$ & $85,10 \%$ & $83,74 \%$ & $84,48 \%$ & $83,71 \%$ & $83,73 \%$ & $85,00 \%$ & $88,92 \%$ & $88,81 \%$ & $89,20 \%$ & $89,45 \%$ & $89,73 \%$ \\
\hline segment & $94,20 \%$ & $93,46 \%$ & $92,03 \%$ & $93,72 \%$ & $94,20 \%$ & $93,03 \%$ & $90,35 \%$ & $90,87 \%$ & $90,26 \%$ & $91,82 \%$ & $92,99 \%$ & $92,08 \%$ & $92,12 \%$ & $93,42 \%$ & $93,07 \%$ \\
\hline sonar & $73,17 \%$ & $71,74 \%$ & $72,71 \%$ & $72,69 \%$ & $73,67 \%$ & $76,00 \%$ & $70,81 \%$ & $72,71 \%$ & $71,29 \%$ & $76,26 \%$ & $75,02 \%$ & $77,00 \%$ & $74,14 \%$ & $75,57 \%$ & $77,50 \%$ \\
\hline spambase & $92,81 \%$ & $92,41 \%$ & $92,11 \%$ & $92,72 \%$ & $92,76 \%$ & $92,26 \%$ & $91,87 \%$ & $91,87 \%$ & $92,05 \%$ & $92,37 \%$ & $93,02 \%$ & $92,65 \%$ & $93,22 \%$ & $93,18 \%$ & $93,41 \%$ \\
\hline spectheart & $72,50 \%$ & $66,25 \%$ & $71,25 \%$ & $68,75 \%$ & $68,75 \%$ & $68,75 \%$ & $72,50 \%$ & $70,00 \%$ & $70,00 \%$ & $71,25 \%$ & $70,00 \%$ & $67,50 \%$ & $70,00 \%$ & $68,75 \%$ & $68,75 \%$ \\
\hline texture & $87,05 \%$ & $87,85 \%$ & $87,05 \%$ & $87,56 \%$ & $88,89 \%$ & $86,89 \%$ & $86,42 \%$ & $86,45 \%$ & $87,24 \%$ & $89,16 \%$ & $95,91 \%$ & $95,69 \%$ & $95,84 \%$ & $96,09 \%$ & $96,31 \%$ \\
\hline thyroid & $99,25 \%$ & $99,08 \%$ & $99,25 \%$ & $99,22 \%$ & $99,25 \%$ & $99,17 \%$ & $99,07 \%$ & $99,07 \%$ & $99,17 \%$ & $99,18 \%$ & $98,69 \%$ & $98,50 \%$ & $98,54 \%$ & $98,63 \%$ & $98,78 \%$ \\
\hline tic-tac-toe & $83,51 \%$ & $84,34 \%$ & $85,90 \%$ & $85,70 \%$ & $88,93 \%$ & $98,02 \%$ & $97,49 \%$ & $97,60 \%$ & $97,70 \%$ & $97,81 \%$ & $98,64 \%$ & $93,73 \%$ & $97,29 \%$ & $98,85 \%$ & $98,43 \%$ \\
\hline titanic & $77,60 \%$ & $77,46 \%$ & $77,87 \%$ & $77,51 \%$ & $77,92 \%$ & $77,60 \%$ & $77,46 \%$ & $77,96 \%$ & $77,92 \%$ & $77,92 \%$ & $77,60 \%$ & $77,65 \%$ & $77,96 \%$ & $77,19 \%$ & $78,01 \%$ \\
\hline twonorm & $80,11 \%$ & $80,04 \%$ & $80,19 \%$ & $80,22 \%$ & $82,82 \%$ & $84,89 \%$ & $83,65 \%$ & $84,18 \%$ & $83,95 \%$ & $86,07 \%$ & $94,11 \%$ & $94,03 \%$ & $93,91 \%$ & $93,84 \%$ & $95,03 \%$ \\
\hline vehicle & $70,34 \%$ & $69,25 \%$ & $69,40 \%$ & $68,45 \%$ & $70,68 \%$ & $64,88 \%$ & $57,68 \%$ & $60,88 \%$ & $60,05 \%$ & $60,88 \%$ & $68,20 \%$ & $67,60 \%$ & $68,08 \%$ & $70,09 \%$ & $69,38 \%$ \\
\hline vowel & $98,08 \%$ & $97,77 \%$ & $97,98 \%$ & $98,28 \%$ & $98,18 \%$ & $97,98 \%$ & $98,28 \%$ & $97,87 \%$ & $98,18 \%$ & $98,18 \%$ & $97,57 \%$ & $96,36 \%$ & $97,67 \%$ & $97,47 \%$ & $97,37 \%$ \\
\hline wisconsin & $94,99 \%$ & $94,28 \%$ & $94,85 \%$ & $94,99 \%$ & $94,99 \%$ & $95,99 \%$ & $95,56 \%$ & $94,70 \%$ & $95,27 \%$ & $95,27 \%$ & $97,42 \%$ & $96,42 \%$ & $96,99 \%$ & $96,70 \%$ & $96,70 \%$ \\
\hline wine & $90,39 \%$ & $88,79 \%$ & $88,24 \%$ & $88,79 \%$ & $90,49 \%$ & $91,57 \%$ & $88,20 \%$ & $85,39 \%$ & $90,36 \%$ & $88,73 \%$ & $94,87 \%$ & $94,97 \%$ & $95,52 \%$ & $95,52 \%$ & $95,52 \%$ \\
\hline yeast & $75,35 \%$ & $74,66 \%$ & $75,20 \%$ & $75,27 \%$ & $75,60 \%$ & $76,08 \%$ & $73,91 \%$ & $75,34 \%$ & $74,93 \%$ & $76,27 \%$ & $76,08 \%$ & $73,85 \%$ & $75,40 \%$ & $75,34 \%$ & $75,40 \%$ \\
\hline zoo & $95,00 \%$ & $90,09 \%$ & $91,09 \%$ & $93,00 \%$ & $92,00 \%$ & $87,09 \%$ & $87,09 \%$ & $85,09 \%$ & $87,09 \%$ & $87,09 \%$ & $93,01 \%$ & $90,18 \%$ & $92,09 \%$ & $92,09 \%$ & $93,09 \%$ \\
\hline
\end{tabular}

Table 7: Classification accuracy (labeled ratio 40\%). 


\begin{tabular}{|c|c|c|c|c|c|c|c|c|c|c|c|c|}
\hline \multirow[b]{2}{*}{ SSL Algorithm } & \multicolumn{3}{|c|}{$10 \%$} & \multicolumn{3}{|c|}{$20 \%$} & \multicolumn{3}{|c|}{$30 \%$} & \multicolumn{3}{|c|}{$40 \%$} \\
\hline & $\mathrm{C} 4.5$ & JRip & $k \mathrm{NN}$ & $\mathrm{C} 4.5$ & JRip & $k \mathrm{NN}$ & $\mathrm{C} 4.5$ & JRip & $k \mathrm{NN}$ & $\mathrm{C} 4.5$ & JRip & $k \mathrm{NN}$ \\
\hline Self-Train & 11 & 9 & 8 & 9 & 6 & 7 & 1 & 5 & 4 & 0 & 5 & 1 \\
\hline Co-Train & 4 & 5 & 2 & 2 & 6 & 4 & 3 & 5 & 4 & 3 & 3 & 2 \\
\hline Tri-Train & 4 & 3 & 8 & 2 & 4 & 7 & 7 & 5 & 13 & 3 & 4 & 8 \\
\hline Supervised & 4 & 4 & 0 & 4 & 5 & 2 & 4 & 5 & 4 & 7 & 8 & 4 \\
\hline EnSSL & 14 & 18 & 11 & 20 & 14 & 15 & 21 & 16 & 9 & 19 & 16 & 17 \\
\hline
\end{tabular}

Table 8: Total wins of each SSL algorithm.

The statistical comparison of multiple algorithms over multiple data sets is fundamental in machine learning and usually it is typically carried out by means of a nonparametric statistical test. Therefore, the Friedman Aligned-Ranks (FAR) test [8] is utilized in order to conduct a complete performance comparison between all algorithms for all the different labeled ratios. Its application will allow us to highlight the existence of significant differences between the proposed algorithm and the classical SSL algorithms and evaluate the rejection of the hypothesis that all the classifiers perform equally well for a given level. Notice that FAR test is considered to be one of the most well-known tools for multiple statistical comparison tests when comparing more than two methods [10]. Furthermore, the Finner test is applied as a post hoc procedure to find out which algorithms present significant differences.

\begin{tabular}{c|cccc}
\hline \multirow{2}{*}{ Ratio } & Classifier & Friedman & \multicolumn{2}{c}{ Finner post-hoc test } \\
& $(\mathrm{C} 4.5)$ & Ranking & $p$-value & Null Hypothesis \\
\hline \multirow{5}{*}{$10 \%$} & EnSSL & 58.4375 & & \\
& Self-training & 76.625 & 0.049750 & rejected \\
& Tri-training & 94.7875 & 0.037739 & rejected \\
& Co-training & 128.225 & 0.025321 & rejected \\
& Supervised & 144.425 & 0.012741 & rejected \\
\hline \multirow{5}{*}{$20 \%$} & EnSSL & 56.6 & & \\
& Self-training & 83.8 & 0.045583 & rejected \\
& Tri-training & 103.85 & 0.037739 & rejected \\
& Supervised & 115.4875 & 0.025321 & rejected \\
& Co-training & 142.7625 & 0.012741 & rejected \\
\hline \multirow{5}{*}{$30 \%$} & EnSSL & 57.575 & & \\
& Tri-training & 93.5375 & 0.044582 & rejected \\
& Supervised & 108.85 & 0.037739 & rejected \\
& Self-training & 109.2625 & 0.025321 & rejected \\
& Co-training & 133.275 & 0.012741 & rejected \\
\hline
\end{tabular}

(continued).

\begin{tabular}{c|cccc}
\hline \multirow{2}{*}{ Ratio } & $\begin{array}{c}\text { Classifier } \\
(\mathrm{C} 4.5)\end{array}$ & $\begin{array}{c}\text { Friedman } \\
\text { Ranking }\end{array}$ & \multicolumn{2}{c}{ Finner post-hoc test } \\
& EnSSL & 58.475 & & Null Hypothesis \\
\hline \multirow{7}{*}{$40 \%$} & Supervised & 77.45 & 0.142611 & accepted \\
& Tri-training & 106.9625 & 0.000239 & rejected \\
& Co-training & 116.2 & 0.000016 & rejected \\
& Self-training & 143.4125 & 0.000000 & rejected \\
\hline
\end{tabular}

Table 9: FAR test and Finner post hoc test (C4.5).

Tables 9, 10 and 11 present the information of the statistical analysis performed by nonparametric multiple comparison procedures for each base learner. The best(lowest) ranking obtained in each FAR test determines the control algorithm for the post hoc test. Moreover, the adjusted $p$ value with Finner's test (Finner APV) is presented based on the control algorithm, at $\alpha=0.05$ level of significance. Clearly, the proposed algorithm exhibits the best overall performance, outperforming the rest SSL algorithms, since it reports the highest probability-based ranking, presenting statistically better results, relative to all labeled ratio.

\section{Conclusions \& future research}

In this work, a new ensemble semi-supervised algorithm is proposed based on a voting methodology. The proposed algorithm combines the individual predictions of three SSL algorithms: Co-training, Self-training and Tri-training via a maximum-probability voting scheme. The numerical experiments and the presented statistical analysis indicate that the proposed algorithm EnSSL outperforms its component SSL algorithms, confirming its efficacy.

An interesting direction for future work is the development of a parallel implementation of the the proposed algorithm. Notice that the implementation of each one of its component based learners in parallel machines constitutes a significant aspect to be studied, since a huge amount of 


\begin{tabular}{c|cccc}
\hline \multirow{2}{*}{ Ratio } & Classifier & Friedman & \multicolumn{2}{c}{ Finner post-hoc test } \\
& (JRip) & Ranking & $p$-value & Null Hypothesis \\
\hline \multirow{7}{*}{$10 \%$} & EnSSL & 62.2625 & & \\
& Self-training & 81.5375 & 0.136404 & accepted \\
& Tri-training & 100.2625 & 0.004429 & rejected \\
& Co-training & 121.0125 & 0.136404 & rejected \\
& Supervised & 137.425 & 0.000000 & rejected \\
\hline \multirow{7}{*}{$20 \%$} & EnSSL & 69.25 & & \\
& Self-training & 95.225 & 0.044749 & rejected \\
& Tri-training & 102.35 & 0.014031 & rejected \\
& Supervised & 116.7 & 0.000492 & rejected \\
& Co-training & 118.975 & 0.000488 & rejected \\
\hline \multirow{5}{*}{$30 \%$} & EnSSL & 66.225 & & \\
& Supervised & 99.9625 & 0.009140 & rejected \\
& Tri-training & 104.175 & 0.004484 & rejected \\
& Self-training & 109.25 & 0.001771 & rejected \\
& Co-training & 122.8875 & 0.000048 & rejected \\
\hline \multirow{7}{*}{$40 \%$} & EnSSL & 64.925 & & \\
& Supervised & 76.1 & 0.387887 & accepted \\
& Tri-training & 107.875 & 0.001206 & rejected \\
& Co-training & 121.175 & 0.000028 & rejected \\
& Self-training & 132.425 & 0.000001 & rejected \\
\hline
\end{tabular}

Table 10: FAR test and Finner post hoc test (JRip).

data can be processed in significantly less computational time. Since the experimental results are quite encouraging, a next step could be the evaluation of the proposed algorithm in specific scientific fields applying real world datasets, such as the educational, health care, etc.

\section{References}

[1] David W. Aha. Lazy Learning. Dordrecht: Kluwer Academic Publishers, 1997. https://doi.org/10.1007/978-94-017-2053-3

[2] Jesús Alcalá-Fdez, Alberto Fernández, Julián Luengo, Joaquín Derrac, Salvador García, Luciano Sánchez, and Francisco Herrera. Keel data-mining software tool: data set repository, integration of algorithms and experimental analysis framework. Journal of Multiple-Valued Logic \& Soft Computing, 17, 2011. https://doi.org/10.1109/nwesp.2011.6088224

[3] Ethem Alpaydin. Introduction to Machine Learning. MIT Press, Cambridge, 2nd edition, 2010. https://doi.org/10.1017/s0269888906220745

[4] Avrim Blum and Tom Mitchell. Combining labeled and unlabeled data with co-training.

\begin{tabular}{c|cccc}
\hline \multirow{2}{*}{ Ratio } & Classifier & Friedman & \multicolumn{2}{c}{ Finner post-hoc test } \\
& $(k \mathrm{NN})$ & Ranking & $p$-value & Null Hypothesis \\
\hline \multirow{7}{*}{$10 \%$} & EnSSL & 59.65 & & \\
& Tri-training & 73.825 & 0.273404 & accepted \\
& Self-training & 89.3375 & 0.028959 & rejected \\
& Co-training & 129.8375 & 0.00000 & rejected \\
& Supervised & 149.85 & 0.00000 & accepted \\
\hline \multirow{7}{*}{$20 \%$} & EnSSL & 59.5125 & & \\
& Tri-training & 79.1625 & 0.128941 & accepted \\
& Self-training & 103.55 & 0.00089 & rejected \\
& Co-training & 130.075 & 0.00000 & rejected \\
& Supervised & 130.2 & 0.00000 & accepted \\
\hline \multirow{7}{*}{$30 \%$} & EnSSL & 70.9625 & & \\
& Tri-training & 86.9875 & 0.045642 & rejected \\
& Supervised & 101.175 & 0.026013 & rejected \\
& Self-training & 117.8625 & 0.000581 & rejected \\
& Co-training & 125.5125 & 0.0001 & rejected \\
\hline \multirow{7}{*}{$40 \%$} & EnSSL & 61.9875 & & \\
& Supervised & 74.3375 & 0.33996 & accepted \\
& Tri-training & 92.2625 & 0.02568 & rejected \\
& Co-training & 124.225 & 0.000003 & rejected \\
& Self-training & 149.6875 & 0.000000 & rejected \\
\hline
\end{tabular}

Table 11: FAR test and Finner post hoc test $(k \mathrm{NN})$.

In 11th annual conference on Computational learning theory, pages 92-100. ACM, 1998. https://doi.org/10.1109/icdm.2001.989574

[5] William W. Cohen. Fast effective rule induction. In International Conference on Machine Learning, pages 115-123, 1995. https://doi.org/10.1016/b9781-55860-377-6.50023-2

[6] Bozidara Cvetkovic, Boštjan Kaluza, Mitja Luštrek, and Matjaz Gams. Semi-supervised learning for adaptation of human activity recognition classifier to the user. In Proceddings of International Joint Conference on Artificial Intelligence, pages 24-29, 2011.

[7] Asif Ekbal and Sivaji Bandyopadhyay. Named entity recognition using appropriate unlabeled data, postprocessing and voting. Informatica, 34(1), 2010.

[8] Helmut Finner. On a monotonicity problem in stepdown multiple test procedures. Journal of the American Statistical Association, 88(423):920-923, 1993. https://doi.org/10.2307/2290782

[9] Matjaž Gams. Weak intelligence: through the principle and paradox of multiple knowledge. Nova Science, 2001. 
[10] Salvador García, Alberto Fernández, Julián Luengo, and Francisco Herrera. Advanced nonparametric tests for multiple comparisons in the design of experiments in computational intelligence and data mining: Experimental analysis of power. Information Sciences, 180(10):2044-2064, 2010. https://doi.org/10.1016/j.ins.2009.12.010

[11] Hristijan Gjoreski, Boštjan Kaluža, Matjaž Gams, Radoje Milić, and Mitja Luštrek. Context-based ensemble method for human energy expenditure estimation. Applied Soft Computing, 37:960-970, 2015. https://doi.org/10.1016/j.asoc.2015.05.001

[12] Tao Guo and Guiyang Li. Improved tri-training with unlabeled data. Software Engineering and Knowledge Engineering: Theory and Practice, pages 139-147, 2012. https://doi.org/10.1007/978-3-642-25349-2_19

[13] Mark Hall, Eibe Frank, Geoffrey Holmes, Bernhard Pfahringer, Peter Reutemann, and Ian H. Witten. The WEKA data mining software: An update. SIGKDD Explorations Newsletters, 11:10-18, 2009. https://doi.org/10.1145/1656274.1656278

[14] Kyaw Kyaw Htike. Hidden-layer ensemble fusion of MLP neural networks for pedestrian detection. Informatica, 41(1), 2017.

[15] Ludmila I. Kuncheva. Combining Pattern Classifiers: Methods and Algorithms. McGraw Hill, John Wiley \& Sons, Inc., second edition, 2014. https://doi.org/10.1002/9781118914564

[16] Jurica Levatić, Sašo Džeroski, Fran Supek, and Tomislav Šmuc. Semi-supervised learning for quantitative structure-activity modeling. Informatica, 37(2), 2013.

[17] Ming Li and Zhi-Hua Zhou. Improve computeraided diagnosis with machine learning techniques using undiagnosed samples. IEEE Transactions on Systems, Man, and Cybernetics-Part A: Systems and Humans, 37(6):1088-1098, 2007. https://doi.org/10.1109/tsmca.2007.904745

[18] Chang Liu and Pong C. Yuen. A boosted co-training algorithm for human action recognition. IEEE transactions on circuits and systems for video technology, 21(9):1203-1213, 2011. https://doi.org/10.1109/tcsvt.2011.2130270

[19] Ioannis E. Livieris, Ioannis Dimopoulos, Thedore Kotsilieris, and Panagiotis Pintelas. Predicting length of stay in hospitalized patients using ssl algorithms. In ACM 8th International Conference on Software
Development and Technologies for Enhancing Accessibility and Fighting Infoexclusion, pages 1-8, 2018. https://doi.org/10.1145/3218585.3218588

[20] Ioannis E. Livieris, Konstantina Drakopoulou, Vassilis Tampakas, Tassos Mikropoulos, and Panagiotis Pintelas. Predicting secondary school students' performance utilizing a semi-supervised learning approach. Journal of Educational Computing Research, 2018. https://doi.org/10.1177/0735633117752614

[21] Ioannis E. Livieris, Konstantina Drakopoulou, Vassilis Tampakas, Tassos Mikropoulos, and Panagiotis Pintelas. Research on e-Learning and ICT in Education, chapter An ensemble-based semi-supervised approach for predicting students' performance, page 25-42. Springer, 2018. https://doi.org/10.1007/9783-319-95059-4_2

[22] Ioannis E. Livieris, Andreas Kanavos, Vassilis Tampakas, and Panagiotis Pintelas. An ensemble SSL algorithm for efficient chest x-ray image classification. Journal of Imaging, 4(7), 2018. https://doi.org/10.3390/jimaging4070095

[23] Ioannis E. Livieris, Tassos Mikropoulos, and Panagiotis Pintelas. A decision support system for predicting students' performance. Themes in Science and Technology Education, 9:43-57, 2016.

[24] Christopher J. Merz. Using correspondence analysis to combine classifiers. Machine Learning, 36:33-58, 1999. https://doi.org/10.1023/A:1007559205422

[25] Vincent $\mathrm{Ng}$ and Claire Cardie. Weakly supervised natural language learning without redundant views. In Proceedings of the 2003 Conference of the North American Chapter of the Association for Computational Linguistics on $\mathrm{Hu}$ man Language Technology-Volume 1, pages 94-101. Association for Computational Linguistics, 2003. https://doi.org/10.3115/1073445.1073468

[26] J. Ross Quinlan. C4.5: Programs for machine learning. Morgan Kaufmann, San Francisco, 1993. https://doi.org/10.1007/BF00993309

[27] Matteo $\mathrm{Re}$ and Giorgio Valentini. Advances in Machine Learning and Data Mining for Astronomy, chapter Ensemble methods: A review, pages 563-594. Chapman \& Hall, 2012. https://doi.org/10.1201/b11822-34

[28] Lior Rokach. Pattern Classification Using Ensemble Methods. World Scientific Publishing Company, 2010. https://doi.org/10.1142/7238 
[29] Moumita Roy, Susmita Ghosh, Ashish Ghosh. A novel approach for change detection of remotely sensed images using semi-supervised multiple classifier system. Information Sciences, 269:35-47, 2014. https://doi.org/10.1016/j.ins.2014.01.037

[30] S.K. Satapathy, A.K. Jagadev, and S. Dehuri. An empirical analysis of different machine learning techniques for classification of EEG signal to detect epileptic seizure. Informatica, 41(1), 2017.

[31] Sandeep Kumar Satapathy, Alok Kumar Jagadev, and Satchidananda Dehuri. Weighted majority voting based ensemble of classifiers using different machine learning techniques for classification of EEG signal to detect epileptic seizure. Informatica, 41(1):99, 2017.

[32] Gasper Slapničar, Mitja Luštrek, and Matej Marinko. Continuous blood pressure estimation from PPG signal. Informatica, 42(1), 2018.

[33] Shiliang Sun and Feng Jin. Robust co-training. International Journal of Pattern Recognition and Artificial Intelligence, 25(07):1113-1126, 2011. https://doi.org/10.1142/s0218001411008981

[34] Shiliang Sun and Qingjiu Zhang. Multipleview multiple-learner semi-supervised learning. Neural processing letters, 34(3):229, 2011. https://doi.org/10.1007/s11063-011-9195-8

[35] Isaac Triguero, Salvador García, and Francisco Herrera. SEG-SSC: A framework based on synthetic examples generation for self-labeled semi-supervised classification. IEEE Transactions on Cybernetics, 45:622-634, 2014. https://doi.org/10.1109/tcyb.2014.2332003

[36] Isaac Triguero, Salvador García, and Francisco Herrera. Self-labeled techniques for semi-supervised learning: taxonomy, software and empirical study. Knowledge and Information Systems, 42(2):245-284, 2015. https://doi.org/10.1007/s10115-013-0706-y

[37] Isaac Triguero, José A. Sáez, Julián Luengo, Salvador García, and Francisco Herrera. On the characterization of noise filters for selftraining semi-supervised in nearest neighbor classification. Neurocomputing, 132:30-41, 2014. https://doi.org/10.1016/j.neucom.2013.05.055

[38] Julius Venskus, Povilas Treigys, Jolita Bernatavičienè, Viktor Medvedev, Miroslav Voznak, Mindaugas Kurmis, and Violeta Bulbenkienè. Integration of a self-organizing map and a virtual pheromone for real-time abnormal movement detection in marine traffic. Informatica, 28(2):359-374, 2017.

[39] Xindong Wu, Vipin Kumar, J. Ross Quinlan, Joydeep Ghosh, Qiang Yang, Hiroshi Motoda, Geoffrey J. McLachlan, Angus Ng, Bing Liu, and Philip S. Yu, Zhi-Hua Zhou, Michael Steinbach, David J. Hand, and Dan Steinberg Top 10 algorithms in data mining. Knowledge and information systems, 14(1):1-37, 2008. https://doi.org/10.1201/9781420089653

[40] Qian Xu, Derek Hao Hu, Hong Xue, Weichuan Yu, and Qiang Yang. Semi-supervised protein subcellular localization. BMC bioinformatics, 10(1):S47, 2009. https://doi.org/10.1186/1471-2105-10-s1-s47

[41] David Yarowsky. Unsupervised word sense disambiguation rivaling supervised methods. In Proceedings of the 33rd annual meeting of the association for computational linguistics, pages 189-196, 1995. https://doi.org/10.3115/981658.981684

[42] Yan Zhou and Sally Goldman. Democratic co-learning. In 16th IEEE International Conference on Tools with Artificial Intelligence (ICTAI), pages 594-602. IEEE, 2004. https://doi.org/10.1109/ictai.2004.48

[43] Zhi-Hua Zhou. When semi-supervised learning meets ensemble learning. In Frontiers of Electrical and Electronic Engineering in China, volume 6, pages 616. Springer, 2011. https://doi.org/10.1007/s11460011-0126-2

[44] Zhi-Hua Zhou and Ming Li. Tri-training: Exploiting unlabeled data using three classifiers. IEEE Transactions on Knowledge and Data Engineering, 17(11):1529-1541, 2005. https://doi.org/10.1109/tkde.2005.186

[45] Xiaojin Zhu. Semi-supervised learning. In Encyclopedia of Machine Learning, pages 892-897. Springer, 2011.

[46] Xiaojin Zhu and Andrew B. Goldberg Introduction to semi-supervised learning. Synthesis lectures on artificial intelligence and machine learning, 3(1):1-130, 2009. https://doi.org/10.2200/s00196ed1v01y200906aim006 